\title{
Comparison of growth indices of Nigella sativa I. under different plant densities and fertilization
}

\author{
loannis Roussis, loanna Kakabouki, Dimitrios Bilalis* \\ Agricultural University of Athens, School of Agriculture, Engineering and Environmental Sciences, Department of Crop Science, Laboratory of \\ Agronomy, 75 lera Odos Str., 11855 Athens, Greece
}

\section{A B S T R A C T}

\begin{abstract}
Agronomic practices such as plant density and fertilizer management are referred to comprise crop environment, which influences plant growth, productivity, and ultimately the yield. The objective of the current study was to evaluate the influence of plant density and fertilization on the growth and growth indices of Nigella sativa crop and to determine the association between yield and growth characteristics at both the single plant and crop stand level. The 2-year experiment was laid out in a split-plot design, with three replications, two main plots (200 and 300 plants $\mathrm{m}^{-2}$ ) and four sub-plots (fertilization treatments: control, compost, farmyard manure and inorganic fertilizer). The highest absolute growth rate (AGR) $\left(0.0321 \mathrm{~g} \mathrm{day}^{-1}\right)$ and relative growth rate (RGR) $\left(0.0714 \mathrm{~g} \mathrm{~g}^{-1} \mathrm{day}^{-1}\right)$ values were recorded when plants subjected to low-density and inorganic fertilization, while the highest crop growth rate (CGR) $\left(8.0342 \mathrm{~g} \mathrm{~m}^{-2} \mathrm{day}^{-1}\right)$ was obtained under high-plant density and inorganic fertilization. Concerning specific leaf area (SLA), the highest value $\left(196.28 \mathrm{~cm}^{2} \mathrm{~g}^{-1}\right)$ was found in inorganic fertilized treatment. Leaf area index (LAI), Leaf area duration (LAD) and Biomass duration (BMD) were positively affected by both plant density and fertilization with the greatest values observed under high-density and fertilization. In conclusion, plant densities higher than 200 plants $\mathrm{m}^{-2}$ lead to higher crop growth, but lower growth of individual plants and decreased seed yield, while the application of inorganic fertilizers increases crop growth and yield as these fertilizers contain higher levels of nitrogen with high solubility and therefore quick availability for the crop than the organic fertilizers.
\end{abstract}

Keywords: Compost; Crop growth rate; Growth parameters; Net assimilation rate; Specific leaf area

\section{INTRODUCTION}

Nigella sativa L., an annual flowering plant belonging to the Ranunculaceae family, is cultivated in Egypt, Iran, India, Saudi Arabia, Syria, Pakistan, Turkey (Riaz et al., 1996) and eastern Mediterranean region to South and West Asia (Tuncturk et al., 2012). N. sativa has been used as a medicinal plant, which may contribute to the improvement of human's health (Riaz et al., 1996; Ahmad et al., 2013). N. sativa seeds contain 30-35\% fixed and $0.5-1.5 \%$ essential oil considered as new sources of oils for pharmaceutical and food industries (Ashraf et al., 2006; Piras et al., 2013). One of the most important compounds of essential oil is thymoquinone, which is classified as terpenoids constitute and imports the plant under investigation about the ability to influence on important human diseases such as cancer (Banerjee et al., 2010) or the metabolic syndrome (Razavi and Hosseinzadeh, 2014).
Agronomic practices such as seed rate, plant density, and fertilizer management are referred to comprise crop environment, which influences plant growth, productivity, and ultimately the yield (Roussis et al., 2017). High density is undesirable because it enhances interplants competition for resources. A previous study has found that optimization of plant density exerts a remarkable influence on crop productivity leading to play a pivotal role in most cultivation practices (Hiltbrunner et al., 2007). In addition, the adequate plant density optimizes the canopy microenvironment, enhances photosynthetic capacity and considerably increases aboveground biomass accumulation and contributes to a higher biomass production (Dai et al., 2015; Yao et al., 2015).

Biomass accumulation and its rate assigned to different plant parts reveal crop growth and development and consequently constitute consequential qualitative physiological parameters. In general, the yield is affected

\footnotetext{
*Corresponding author:

Dimitrios Bilalis, Agricultural University of Athens, School of Agriculture, Engineering and Environmental Sciences, Department of Crop Science, Laboratory of Agronomy, 75 lera Odos Str., 11855 Athens, Greece. E-mail: bilalisdimitrios@gmail.com
}

Received: 21 December 2018; $\quad$ Accepted: 14 March 2019 
by the biomass and photosynthate redistribution after heading and blossoming stages (Weraduwage et al., 2015). The biomass accumulation more than a given quantity normally results in high yields. For instance, in cereal crops, it has been observed that seed yield is highly associated with biomass accumulation to seeds (Dordas, 2009). As a consequence, for higher seed yields, biomass accumulation throughout the vegetative phase should be enhanced first, succeeded by higher biomass allocation to seeds (Zhang and Flottmann, 2016).

Plant growth is defined as an irreversible permanent increase in size over time, accompanied by an increase in dry weight, indicating gradual and progressive structural and physiological changes and development and can be identified by mathematical models that contribute synthesizing and improving knowledge about a productive system (Montenegro et al., 2017). Plant growth analysis is an explanatory and integrative approach using observed primary data, in particular weights, areas, volumes, and contents of plants to evaluate the processes involving the whole plant or plant population (Hunt, 1990). This approach allows evaluating possible strategies for agronomic management and estimating potential yield in regard to environmental conditions and management practices (Lambers et al., 1998). Plant growth parameters, in particular leaf area ratio (LAR), net assimilation rate (NAR), and relative growth rate (RGR), describe plant morphophysiological responses at different timespans and allow to track dynamics of photosynthetic production, evaluated by dry biomass accumulation (Sugár et al., 2017).

Several research articles have demonstrated the influence of various agronomic practices on growth of several crops, including wheat (Sugár et al., 2017), maize (Bullock et al., 1993) and sugar beet (Hoffmann and Kluge-Severin, 2011). However, there was no information available about the growth analysis of $N$. sativa crop, especially under Mediterranean semi-arid conditions. The current study was aimed to investigate the effect of different plant densities and fertilization on the growth and growth indices of $N$. sativa crop and to determine the association between yield and growth characteristics at both the single plant and crop stand level.

\section{MATERIALS AND METHODS}

\section{Site description and experimental design}

A Nigella sativa crop was established in the organic experimental field of the Agricultural University of Athens (Latitude: 3759' 1.70" N, Longitude: 23²4' 7.04" E, Altitude: $29 \mathrm{~m}$ above sea level) during 2017 and 2018 growing seasons. The soil was a clay loam $(29.8 \%$ clay, $34.3 \%$ silt and $35.9 \%$ sand) with $\mathrm{pH}\left(1: 1 \mathrm{H}_{2} \mathrm{O}\right) 7.34$, nitratenitrogen $\left(\mathrm{NO}_{3}-\mathrm{N}\right) 12.4 \mathrm{mg} \mathrm{kg}^{-1}$ soil, available phosphorus (P) $13.2 \mathrm{mg} \mathrm{kg}^{-1}$ soil, available potassium (K) $201 \mathrm{mg} \mathrm{kg}^{-1}$ soil, $15.99 \% \mathrm{CaCO}_{3}$ and $1.82 \%$ organic matter. The site was managed according to the organic agricultural guidelines (EC 834/2007).

The experiment was set up on an area of $302 \mathrm{~m}^{2}$ according to the split plot design with two main plots (plant densities: 200 plants $\mathrm{m}^{-2}$ and 300 plants $\mathrm{m}^{-2}$ ), four sub-plots [fertilization treatments: control (untreated), seaweed compost $\left(2000 \mathrm{~kg} \mathrm{ha}^{-1}\right.$ Posidonia 1-2\% N, Compost Hellas S.A.), farmyard manure $\left(2000 \mathrm{~kg} \mathrm{ha}^{-1}\right.$, solid, $1.52 \% \mathrm{~N})$, and inorganic fertilizer $\left(300 \mathrm{~kg} \mathrm{ha}^{-1}\right.$ Enpeka 15-15-15+5 S, Compo GmbH)], and three replications for each treatment. The main plot and subplot sizes were $42.25 \mathrm{~m}^{2}(6.5 \mathrm{~m} \times 6.5 \mathrm{~m})$ and $9 \mathrm{~m}^{2}(3 \mathrm{~m}$ $\times 3 \mathrm{~m}$ ), respectively. The soil was prepared by ploughing at a depth of about $0.25 \mathrm{~m}$. Fertilizers were applied by hand on the soil surface and then harrowed. N. sativa was sown by hand in rows $30 \mathrm{~cm}$ apart at a depth of $0.5-1 \mathrm{~cm}$. Seed sowing was performed on $1^{\text {st }}$ February in both years. Emergence was on $25^{\text {th }}$ and $19^{\text {th }}$ February in 2017 and 2018, respectively. Seedlings were thinned at the four-true leaf stage to the examined plant densities, which were 200 and 300 plants $\mathrm{m}^{-2}$. Weeds were controlled by hand-hoeing as and when needed and before canopy closure.

\section{Sampling procedures, measurements and methods}

Five plant samples were randomly collected from each sub-plot at 45, 60, 75, 85, 100 and 115 Days After Sowing (DAS). The plants selected were divided into stems, leaves, flowers, follicles, and seeds. Leaf area was measured using an automatic leaf area meter (Delta-T Devices Ltd, Burwell, Cambridge, UK). Above-ground dry matter was determined after drying for 48 hours at $64^{\circ} \mathrm{C}$. These measurements were used for growth analysis. The growth parameters or indices were calculated using the following formulas according to Hunt (1990):

$$
\begin{aligned}
& \text { LAI (Leaf Area Index })=L c a / P \quad\left(\mathrm{~m}^{2} \mathrm{~m}^{-2}\right) \\
& L A D(\text { Leaf Area Duration })=\left[\left(\mathrm{LAI}_{2}+L A I_{1}\right) / 2\right]\left[t_{2}-t_{1}\right]
\end{aligned}
$$

$$
\begin{array}{ll}
\text { SLW (Specific Leaf Weight) }=\mathrm{Lw} / \mathrm{La} & \left(\mathrm{g} \mathrm{cm}^{-2}\right) \\
\text { LWR (Leaf Weight Ratio })=L w / W & \left(\mathrm{~g} \mathrm{~g}^{-1}\right) \\
\text { SLA (Specific Leaf Area) }=L a / L w & \left(\mathrm{~cm}^{2} \mathrm{~g}^{-1}\right) \\
\text { LAR (Leaf Area Ratio) }=L a / W & \left(\mathrm{~cm}^{2} \mathrm{~g}^{-1}\right)
\end{array}
$$


$B M D$ (Biomass Duration $)=\frac{(W \circ 2-W c 1)(t 2-t 1)}{(\ln W c 2-\ln W \circ 1)}$

(days)

AGR (Absolute Growth Rate) $=\frac{(W 2-W 1)}{(t 2-t 1)}\left(\mathrm{g} \mathrm{day}^{-1}\right)$

ALGR (Absolute Leaf Growth Rate) $=\frac{(L w 2-L w 1)}{(t 2-t 1)}$

$\left(\mathrm{g} \mathrm{day}^{-1}\right)(9)$

RGR (Relative Growth Rate) $=\frac{(\ln W 2-\ln W 1)}{(t 2-t 1)}$

$\left(\mathrm{g} \mathrm{g}^{-1}\right.$ day $\left.^{-1}\right)$

$N A R$ (Net Assimilation Rate) $=\frac{(W 2-W 1)}{(t 2-t 1)}$

$\frac{(\ln \operatorname{La} 2-\ln \operatorname{La} 1)}{(\operatorname{La} 2-\operatorname{La} 1)}$

$\left(\mathrm{g} \mathrm{cm}^{-2}\right.$ day $\left.^{-1}\right)$

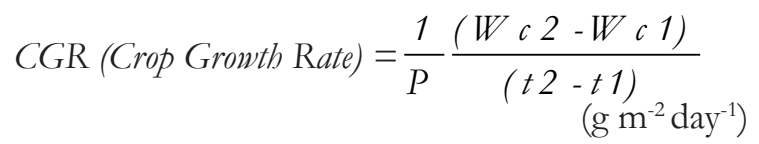

where, La represents total one side area of photosynthetically leaf tissue of a plant $\left(\mathrm{cm}^{2}\right)$; Lca $=$ total one side area of leaf tissue of canopy covering an area of $1 \mathrm{~m}^{2}\left(\mathrm{~m}^{2}\right)$, $\mathrm{P}=$ unit ground surface area $\left(\mathrm{m}^{2}\right) ; \mathrm{t}_{1}$ and $\mathrm{t}_{2}$ represent time points of $N$. sativa growth; $L w$ represents dry matter of photosynthetically leaf tissue (g); W represents total plant dry matter (g); Wc represents total dry matter of canopy covering an area of $1 \mathrm{~m}^{2}(\mathrm{~g})$.

The plants were harvested at physiological maturity on $3^{\text {rd }}$ June 2017 (122 DAS) and $6^{\text {th }}$ June 2018 (125 DAS). The seed yield was determined by plants derived from the middle sub-plot area $\left(1 \mathrm{~m}^{2}\right)$.

\section{Meteorological data, thermal time and phenological stages}

Meteorological data (mean monthly air temperature and precipitation) during the growing periods were obtained from the weather station of the Agricultural University of Athens and are presented in Figure 1. Total precipitation in 2017 and 2018 (from February to May) was 160.2 and $160.8 \mathrm{~mm}$, respectively. The mean temperature throughout the growing season was $16.2^{\circ} \mathrm{C}$ for 2017 and $17.8^{\circ} \mathrm{C}$ for 2018 .

During the growing cycle, heat accumulation in growing degree days (ADD) from sowing until harvest, summing the growing degree days (GDD) in each time period evaluated. Growing Degree Days (GDD) was calculated as:

$G D D_{i}=\sum_{i}^{n}\left[\frac{T_{m a x}+T_{m i n}}{2}\right]-T_{b a s e}$ where, $\mathrm{GDD}_{\mathrm{i}}$ is the accumulated growing degree days, $\mathrm{T}_{\min }$ and $\mathrm{T}_{\max }$ are the minimum and maximum air temperatures, respectively. $\mathrm{T}_{\text {base }}$ also is $N$. sativa base temperature $\left(\mathrm{T}_{\text {base }}=5^{\circ} \mathrm{C}\right)$ (Ghaderi et al., 2008).

For clarity, ADD and the dates of measurements and basic phenological stages of the crop are reported in Table 1 . To record the developmental stages, $1 \mathrm{~m}^{2}$ of each sub-plot was observed. The phenological stages were examined when at least one plant in each sub-plot pointed out that stage. The phenological stages were: emergence, when at least one seedling was detected in the sub-plot; blooming, when a flower bud per plant was detected at a minimum; flowering, when an unfolded flower per plant was detected at a minimum; seed formation, when visible green seeds in follicles of at least one plant were detected in the sub-plot.

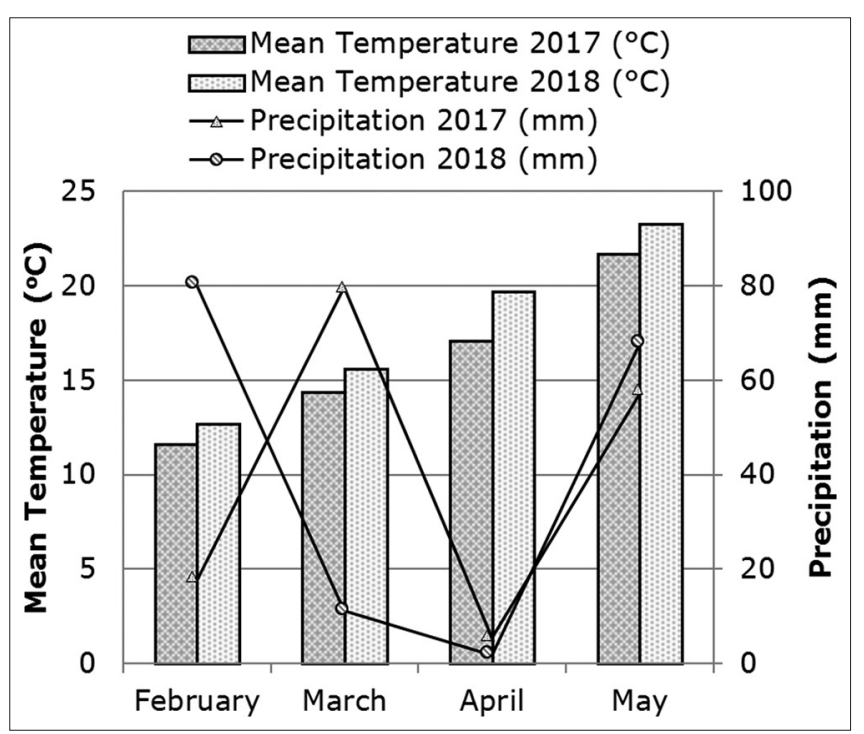

Fig 1. Meteorological data (mean monthly temperature and precipitation) during the growing periods (February-May, 2017 and 2018).

Table 1: Accumulated Degree Days (ADD) and dates of basic phenological stages and measurements (expressed in DAS) obtained during the growing cycle for each year.

\begin{tabular}{lccccc}
\hline Developmental Stage & \multicolumn{2}{c}{2017} & & \multicolumn{2}{c}{2018} \\
\cline { 2 - 3 } \cline { 6 - 6 } or Measurement & DAS & ADD & & DAS & ADD \\
\hline Emergence & 25 & 166.4 & & 19 & 152.9 \\
$1^{\text {st }}$ Measurement & 45 & 330.1 & & 45 & 410.4 \\
$2^{\text {nd }}$ Measurement & 60 & 497.0 & & 60 & 568.7 \\
Blooming & 71 & 622.9 & & 73 & 725.4 \\
$3^{\text {rd }}$ Measurement & 75 & 676.2 & & 75 & 772.9 \\
Flowering & 80 & 730.6 & & 80 & 845.5 \\
$4^{\text {th }}$ Measurement & 85 & 789.7 & & 85 & 927.3 \\
Seed Formation & 88 & 839.4 & & 90 & 1021.5 \\
$5^{\text {th }}$ Measurement & 100 & 1045.4 & & 100 & 1186.1 \\
$6^{\text {th }}$ Measurement & 115 & 1294.0 & & 115 & 1470.5 \\
Physiological & 122 & 1417.3 & & 125 & 1685.2 \\
maturity - Harvest & & & & \\
\hline
\end{tabular}




\section{Statistical analysis}

The experimental data were checked for normality and subjected to statistical analysis using the SigmaPlot 12 statistical software (Systat Software Inc., San Jose, CA, USA). Data of traits produced by plant density and fertilization treatments in the two years were analyzed adopting 2 X 2 X 4 factorial design (two years; two plant density treatments and three fertilization treatments) laid out in a split-plot design with three replications. The Analysis of Variance (ANOVA) used a mixed model, with years and replications as random effects and plant density and fertilization as fixed effects. Differences between means were separated using Tukey's honestly significant difference test (Tukey's HSD). Correlation analyses were used to describe the relationships between growth parameters and yield components using Pearson's correlation. All comparisons were made at the $5 \%$ level of significance $(\mathrm{p} \leq 0.05)$.

\section{RESULTS}

\section{Thermal time and phenology}

The effect of the year associated with accumulated growing degree days (ADD) between phenological events was clearly revealed by the data presented in Table 1 . Since $N$. sativa crop was sown on the same date ( $1^{\text {st }}$ February) during the two experimental years, the second year (2018) resulted in more accumulated ADD from emergence to physiological maturity. There were differences in the speed of seedling emergence among years. In the first year of the experiment, seedlings emerged at 25 DAS (166.4 ADD), while during the second year, seedling emergence was done 6 days earlier at 19 DAS (152.9 ADD). Flowering was not related to ADD, since, N. sativa was flowered on the same date (80 DAS) in both years. The differences in accumulated ADD may be explained by the fact that a higher temperature range $\left(\Delta \mathrm{T}=18.6^{\circ} \mathrm{C}\right)$ was observed in the first year than the second one $\left(\Delta \mathrm{T}=15.3^{\circ} \mathrm{C}\right)$. Maximum temperatures recorded in 2017 during the growing season ranged between 18.9 and $34.5^{\circ} \mathrm{C}$ and minimums between 2.3 (in February 2017) and $14.0^{\circ} \mathrm{C}$. In the second year, maximum temperatures ranged between 18.9 and $29.6^{\circ} \mathrm{C}$ and minimums between 6.3 and $16.0^{\circ} \mathrm{C}$.

\section{Above-ground dry matter, leaf area and leaf dry matter per plant}

The dry matter accumulation was significantly affected by fertilization treatments during seed formation and maturity. In response to fertilizers, the above-ground dry matter increased up to the inorganic fertilization, and the higher weights observed at 115 DAS with the values being 1.579 and $1.393 \mathrm{~g} \mathrm{plant}^{-1}$ in 2017 and 2018, respectively (Table 2).

The combined analysis of variance revealed that the dynamics of leaf area growth per plant was significantly affected by both plant density and fertilization (Table 12). Leaf area per plant was higher in the low-density $\left(200\right.$ plants $\mathrm{m}^{-2}$ ) than in high-density plots $\left(300\right.$ plants $\mathrm{m}^{-2}$ ) during the experimental periods, and the maximum area was

Table 2: Effect of plant density (200 and 300 plants $\mathrm{m}^{-2}$ ) and fertilization (control, manure, compost and inorganic) on above-ground dry matter per plant by Tukey's HSD test.

\begin{tabular}{|c|c|c|c|c|c|c|c|c|c|c|c|c|}
\hline \multirow{4}{*}{ Fertilization } & \multicolumn{12}{|c|}{ Plant Density (plants $\mathrm{m}^{-2}$ ) } \\
\hline & 200 & 300 & 200 & 300 & 200 & 300 & 200 & 300 & 200 & 300 & 200 & 300 \\
\hline & \multicolumn{12}{|c|}{ Above-ground dry matter per plant (g) } \\
\hline & \multicolumn{2}{|c|}{45 DAS } & \multicolumn{2}{|c|}{60 DAS } & \multicolumn{2}{|c|}{75 DAS } & \multicolumn{2}{|c|}{85 DAS } & \multicolumn{2}{|c|}{100 DAS } & \multicolumn{2}{|c|}{115 DAS } \\
\hline \multicolumn{13}{|l|}{2017} \\
\hline Control & 0.198 & 0.194 & 0.479 & 0.471 & 0.822 & 0.783 & 1.024 & 0.971 & 1.069 & 0.994 & 1.148 & 1.083 \\
\hline Manure & 0.212 & 0.180 & 0.568 & 0.482 & 0.970 & 0.814 & 1.228 & 1.023 & 1.310 & 1.112 & 1.417 & 1.183 \\
\hline Compost & 0.225 & 0.199 & 0.649 & 0.536 & 1.160 & 0.949 & 1.490 & 1.208 & 1.559 & 1.300 & 1.690 & 1.393 \\
\hline Inorganic & 0.215 & 0.188 & 0.625 & 0.541 & 1.125 & 0.971 & 1.454 & 1.246 & 1.587 & 1.325 & 1.709 & 1.449 \\
\hline$F_{\text {plant density }}$ & \multicolumn{2}{|c|}{$2.0863^{\text {ns }}$} & \multicolumn{2}{|c|}{$3.0100^{\text {ns }}$} & \multicolumn{2}{|c|}{$3.7980^{\text {ns }}$} & \multicolumn{2}{|c|}{$4.1511^{\mathrm{ns}}$} & \multicolumn{2}{|c|}{$4.0317^{\mathrm{ns}}$} & \multicolumn{2}{|c|}{$3.8102^{\text {ns }}$} \\
\hline$F_{\text {fertilization }}$ & \multicolumn{2}{|c|}{$0.2562^{\text {ns }}$} & \multicolumn{2}{|c|}{$1.7078^{\text {ns }}$} & \multicolumn{2}{|c|}{$2.9553^{\text {ns }}$} & \multicolumn{2}{|c|}{$\begin{array}{c}3.6084^{*} \\
\text { (Tukey }=0.27715)\end{array}$} & \multicolumn{2}{|c|}{$\begin{array}{c}4.0817^{*} \\
\text { (Tukey }=0.39737)\end{array}$} & \multicolumn{2}{|c|}{$\begin{array}{c}3.9409^{*} \\
(\text { Tukey }=0.32733)\end{array}$} \\
\hline $\begin{array}{l}F_{\text {plant density } x \text { fertilization }} \\
2018\end{array}$ & \multicolumn{2}{|c|}{$0.1691^{\text {ns }}$} & \multicolumn{2}{|c|}{$0.2897^{\text {ns }}$} & \multicolumn{2}{|c|}{$0.2522^{\mathrm{ns}}$} & \multicolumn{2}{|c|}{$0.2746^{\mathrm{ns}}$} & \multicolumn{2}{|c|}{$0.1974^{\mathrm{ns}}$} & \multicolumn{2}{|c|}{$0.2202^{\mathrm{ns}}$} \\
\hline Control & 0.173 & 0.172 & 0.436 & 0.419 & 0.735 & 0.697 & 0.890 & 0.846 & 0.958 & 0.915 & 1.022 & 0.956 \\
\hline Manure & 0.183 & 0.158 & 0.499 & 0.426 & 0.872 & 0.861 & 1.108 & 0.910 & 1.161 & 0.979 & 1.262 & 1.026 \\
\hline Compost & 0.197 & 0.179 & 0.439 & 0.492 & 0.992 & 0.843 & 1.274 & 1.080 & 1.343 & 1.166 & 1.452 & 1.251 \\
\hline Inorganic & 0.193 & 0.165 & 0.562 & 0.470 & 1.019 & 0.724 & 1.325 & 1.082 & 1.430 & 1.168 & 1.534 & 1.252 \\
\hline$F_{\text {plant density }}$ & \multicolumn{2}{|c|}{$1.6848^{\text {ns }}$} & \multicolumn{2}{|c|}{$2.3884^{\mathrm{ns}}$} & \multicolumn{2}{|c|}{$3.3928^{\text {ns }}$} & \multicolumn{2}{|c|}{$4.1526^{n s}$} & \multicolumn{2}{|c|}{$3.3518^{\text {ns }}$} & & $75^{\text {ns }}$ \\
\hline$F_{\text {fertilization }}$ & 0.31 & $03^{\text {ns }}$ & 1.34 & $29^{\text {ns }}$ & 2.45 & $28^{\text {ns }}$ & (Tuke & $\begin{array}{l}57^{\star} \\
.25125)\end{array}$ & (Tuke & $\begin{array}{l}332^{*} \\
0.35895)\end{array}$ & (Tuk & $\begin{array}{l}15^{\star} \\
.38823)\end{array}$ \\
\hline$F_{\text {plant density } x \text { fertilization }}$ & 0.21 & $37^{\text {ns }}$ & 0.19 & $74^{\text {ns }}$ & 0.20 & $11^{\text {ns }}$ & & $47^{\text {ns }}$ & & $15^{\text {ns }}$ & & $47^{\text {ns }}$ \\
\hline
\end{tabular}
not significant ( $P>0.05)$. 
found during the flowering stage (85 DAS). Averaged over fertilization treatments and years, the maximum values at 85 DAS were $95.25 \mathrm{~cm}^{2}$ plant $\mathrm{t}^{-1}$ for low density and $75.09 \mathrm{~cm}^{2}$ plant $^{-1}$ for high-density (Table 3 ). The dynamics in the control and manure treatments were quite distinct from that in the compost and inorganic fertilization treatments. Averaged over plant densities and years, the maximum leaf area per plant was lower in the control $\left(55.92 \mathrm{~cm}^{2}\right.$ plant $\left.{ }^{-1}\right)$ and significantly greater in the manure treatment $\left(74.36 \mathrm{~cm}^{2}\right.$ plant $\left.^{-1}\right)$, while the greatest values were obtained in the compost $\left(97.74 \mathrm{~cm}^{2}\right.$ plant $\left.{ }^{-1}\right)$ and inorganic $\left(112.68 \mathrm{~cm}^{2}\right.$ plant $\left.^{-1}\right)$ treatments.

As with the leaf area per plant, the leaf dry matter was also significantly influenced by the different plant densities and fertilization and the greatest values found at 85 DAS

Table 3: Effect of plant density (200 and 300 plants $\mathrm{m}^{-2}$ ) and fertilization (control, manure, compost and inorganic) on leaf area and leaf dry matter per plant by Tukey's HSD test.

\begin{tabular}{|c|c|c|c|c|c|c|c|c|c|c|c|c|}
\hline \multirow{4}{*}{ Fertilization } & \multicolumn{12}{|c|}{ Plant Density (plants $\mathrm{m}^{-2}$ ) } \\
\hline & 200 & 300 & 200 & 300 & 200 & 300 & 200 & 300 & 200 & 300 & 200 & 300 \\
\hline & \multicolumn{12}{|c|}{ Leaf area per plant $\left(\mathrm{cm}^{2}\right)$} \\
\hline & \multicolumn{2}{|c|}{45 DAS } & \multicolumn{2}{|c|}{60 DAS } & \multicolumn{2}{|c|}{75 DAS } & \multicolumn{2}{|c|}{85 DAS } & \multicolumn{2}{|c|}{100 DAS } & \multicolumn{2}{|c|}{115 DAS } \\
\hline \multicolumn{13}{|l|}{2017} \\
\hline Control & 15.71 & 14.53 & 35.89 & 31.05 & 55.82 & 50.51 & 57.29 & 50.89 & 39.60 & 33.51 & 30.37 & 30.05 \\
\hline Manure & 20.36 & 14.85 & 53.66 & 36.69 & 81.98 & 57.52 & 85.09 & 58.57 & 60.91 & 40.92 & 37.28 & 29.83 \\
\hline Compost & 23.49 & 18.76 & 65.48 & 51.63 & 100.70 & 77.36 & 108.65 & 84.43 & 81.29 & 61.47 & 41.59 & 35.90 \\
\hline Inorganic & 23.08 & 19.78 & 70.55 & 58.82 & 112.29 & 91.19 & 118.90 & 98.79 & 90.83 & 72.66 & 37.19 & 33.41 \\
\hline$F_{\text {plant density }}$ & \multicolumn{2}{|c|}{$\begin{array}{c}8.0284^{*} \\
(\text { Tukey=3.3379) }\end{array}$} & \multicolumn{2}{|c|}{$\begin{array}{c}11.0689^{* *} \\
\text { (Tukey=9.562) }\end{array}$} & \multicolumn{2}{|c|}{$\begin{array}{c}11.1163^{* *} \\
\text { (Tukey=16.400) }\end{array}$} & \multicolumn{2}{|c|}{$\begin{array}{c}11.2436^{* *} \\
\text { (Tukey=17.676) }\end{array}$} & \multicolumn{2}{|c|}{$\begin{array}{c}14.3605^{* *} \\
\text { (Tukey=15.427) }\end{array}$} & \multicolumn{2}{|c|}{$2.3751^{\mathrm{ns}}$} \\
\hline$F_{\text {fertilization }}$ & \multicolumn{2}{|c|}{$\begin{array}{c}5.3886^{* *} \\
(\text { Tukey }=5.8214)\end{array}$} & \multicolumn{2}{|c|}{$\begin{array}{c}15.3676^{\star \star \star} \\
\text { (Tukey=16.865) }\end{array}$} & \multicolumn{2}{|c|}{$\begin{array}{c}14.7459^{* * *} \\
\text { (Tukey=26.546) }\end{array}$} & \multicolumn{2}{|c|}{$\begin{array}{c}18.2067^{* \star *} \\
(\text { Tukey }=27.489)\end{array}$} & \multicolumn{2}{|c|}{$\begin{array}{c}23.0326^{* * *} \\
(\text { Tukey }=21.237)\end{array}$} & \multicolumn{2}{|c|}{$1.6158^{\text {ns }}$} \\
\hline $\begin{array}{l}F_{\text {plant density } X \text { fertilization }} \\
2018\end{array}$ & \multicolumn{2}{|c|}{$0.5351^{\text {ns }}$} & \multicolumn{2}{|c|}{$0.5222^{\text {ns }}$} & \multicolumn{2}{|c|}{$0.6452^{\text {ns }}$} & 0.6 & $16^{\text {ns }}$ & & $29^{\text {ns }}$ & & $3^{\text {ns }}$ \\
\hline Control & 16.74 & 15.59 & 38.71 & 32.92 & 60.39 & 53.99 & 61.58 & 53.91 & 41.96 & 35.83 & 32.48 & 32.13 \\
\hline Manure & 21.87 & 15.85 & 56.45 & 38.55 & 87.97 & 60.77 & 91.73 & 62.03 & 64.88 & 43.00 & 39.26 & 31.37 \\
\hline Compost & 23.50 & 20.30 & 65.44 & 55.14 & 101.54 & 83.45 & 108.46 & 89.41 & 80.47 & 67.59 & 41.77 & 38.13 \\
\hline Inorganic & 24.48 & 20.34 & 75.91 & 60.74 & 121.65 & 94.90 & 130.33 & 102.69 & 96.09 & 76.40 & 40.23 & 35.26 \\
\hline$F_{\text {plant density }}$ & $\begin{array}{r}5 . \\
\text { (Tuke }\end{array}$ & $\begin{array}{l}556^{*} \\
=3.5647)\end{array}$ & $\begin{array}{r}8 \\
\text { (Tuke }\end{array}$ & $\begin{array}{l}19^{*} \\
11.785)\end{array}$ & $\begin{array}{r}9.4 \\
\text { (Tuke) }\end{array}$ & $\begin{array}{l}30^{* *} \\
17.697)\end{array}$ & $\begin{array}{r}8.9 \\
\text { (Tukey }\end{array}$ & $\begin{array}{l}80^{* *} \\
18.536)\end{array}$ & $\begin{array}{r}8 \\
\text { (Tuke }\end{array}$ & $\begin{array}{l}86^{*} \\
14.738)\end{array}$ & & 76ns \\
\hline$F_{\text {fertilization }}$ & $\begin{array}{r}3 . \\
\text { (Tuke }\end{array}$ & $\begin{array}{l}913^{*} \\
=4.7948)\end{array}$ & $\begin{array}{r}11 \\
\text { (Tuke }\end{array}$ & $\begin{array}{l}96^{* * *} \\
19.135)\end{array}$ & $\begin{array}{r}12 . \\
\text { (Tuke) }\end{array}$ & $\begin{array}{l}30^{\star \star \star} \\
29.439)\end{array}$ & $\begin{array}{r}13 . \\
\text { (Tuke }\end{array}$ & $31.971)$ & $\begin{array}{r}16 \\
\text { (Tuk }\end{array}$ & $\begin{array}{l}65^{\star \star \star} \\
23.423)\end{array}$ & & ns \\
\hline$F_{\text {plant density } X \text { fertilization }}$ & & $479^{\text {ns }}$ & & $86^{\text {ns }}$ & & $53^{\text {ns }}$ & 0.5 & $96^{\text {ns }}$ & & $97^{\text {ns }}$ & & $7^{\text {ns }}$ \\
\hline & & & & & Le & ry ma & per pla & (g) & & & & \\
\hline & & DAS & & DAS & & AS & 85 & DAS & & DAS & & DAS \\
\hline 2017 & & & & & & & & & & & & \\
\hline Control & 0.137 & 0.132 & 0.245 & 0.219 & 0.319 & 0.290 & 0.370 & 0.332 & 0.280 & 0.244 & 0.182 & 0.158 \\
\hline Manure & 0.171 & 0.127 & 0.333 & 0.240 & 0.450 & 0.322 & 0.528 & 0.372 & 0.382 & 0.273 & 0.247 & 0.174 \\
\hline Compost & 0.183 & 0.148 & 0.361 & 0.294 & 0.548 & 0.419 & 0.629 & 0.496 & 0.445 & 0.350 & 0.278 & 0.223 \\
\hline Inorganic & 0.173 & 0.147 & 0.363 & 0.304 & 0.565 & 0.471 & 0.685 & 0.557 & 0.474 & 0.389 & 0.293 & 0.239 \\
\hline$F_{\text {plant density }}$ & $\begin{array}{r}7 . \\
\text { (Tukey }\end{array}$ & $\begin{array}{l}385^{*} \\
0.02172)\end{array}$ & $\begin{array}{r}9 . \\
\text { (Tuke }\end{array}$ & $\begin{array}{l}21^{* *} \\
0.05163)\end{array}$ & $\begin{array}{r}10 . \\
\text { (Tukey }\end{array}$ & $\begin{array}{l}57^{* *} \\
.09159)\end{array}$ & $\begin{array}{r}9.8 \\
\text { (Tukey }\end{array}$ & $\begin{array}{l}42^{* *} \\
0.11372)\end{array}$ & $\begin{array}{r}10 \\
\text { (Tuke) }\end{array}$ & $\begin{array}{l}95^{* *} \\
0.07370)\end{array}$ & $\begin{array}{r}10 \\
\text { (Tuke) }\end{array}$ & $\begin{array}{l}218^{\star *} \\
0.04371)\end{array}$ \\
\hline$F_{\text {fertilization }}$ & & $277^{\text {ns }}$ & $\begin{array}{r}5 . \\
\text { (Tuke }\end{array}$ & $\begin{array}{l}27^{* *} \\
0.09215)\end{array}$ & $\begin{array}{r}11.2 \\
\text { (Tukey }\end{array}$ & $\begin{array}{l}4^{\star \star *} \\
.13677)\end{array}$ & $\begin{array}{r}10.9 \\
\text { (Tukey= }\end{array}$ & $\begin{array}{l}77^{* * *} \\
0.16785)\end{array}$ & $\begin{array}{r}9 . \\
\text { (Tuke) }\end{array}$ & $\begin{array}{l}81^{* \star \star} \\
0.08683)\end{array}$ & $\begin{array}{r}7 . \\
\text { (Tuke) }\end{array}$ & $\begin{array}{l}63^{\star *} \\
0.07316)\end{array}$ \\
\hline $\begin{array}{l}F_{\text {plant density } X \text { fertilization }} \\
2018\end{array}$ & & $371^{\text {ns }}$ & & $04^{\text {ns }}$ & & $17^{\text {ns }}$ & 0.5 & $17^{\text {ns }}$ & & $16^{\text {ns }}$ & & $97^{\text {ns }}$ \\
\hline Control & 0.145 & 0.141 & 0.261 & 0.232 & 0.347 & 0.310 & 0.395 & 0.355 & 0.299 & 0.260 & 0.194 & 0.165 \\
\hline Manure & 0.180 & 0.134 & 0.356 & 0.250 & 0.479 & 0.339 & 0.562 & 0.394 & 0.411 & 0.283 & 0.263 & 0.184 \\
\hline Compost & 0.182 & 0.158 & 0.369 & 0.309 & 0.543 & 0.459 & 0.629 & 0.537 & 0.443 & 0.381 & 0.279 & 0.238 \\
\hline Inorganic & 0.187 & 0.155 & 0.391 & 0.318 & 0.621 & 0.486 & 0.726 & 0.577 & 0.511 & 0.406 & 0.311 & 0.249 \\
\hline$F_{\text {plant density }}$ & $\begin{array}{r}5 . \\
\text { (Tukey }\end{array}$ & $\begin{array}{l}720^{*} \\
0.02378)\end{array}$ & $\begin{array}{r}8 \\
\text { (Tuke }\end{array}$ & $\begin{array}{l}282^{*} \\
0.05686)\end{array}$ & $\begin{array}{r}7.8 \\
\text { (Tukey }\end{array}$ & $\begin{array}{l}61^{*} \\
.09001)\end{array}$ & $\begin{array}{r}8.2 \\
\text { (Tukey= }\end{array}$ & $\begin{array}{l}52^{*} \\
0.10995)\end{array}$ & $\begin{array}{r}8 \\
\text { (Tuke) }\end{array}$ & $\begin{array}{l}28^{*} \\
0.07981)\end{array}$ & $\begin{array}{r}8 . \\
\text { (Tuke) }\end{array}$ & $\begin{array}{l}47^{*} \\
0.04767)\end{array}$ \\
\hline$F_{\text {fertilization }}$ & & $283^{\text {ns }}$ & $\begin{array}{r}4 \\
\text { (Tuke }\end{array}$ & $\begin{array}{l}129^{*} \\
0.10509)\end{array}$ & $\begin{array}{r}8.1 \\
\text { (Tukey }\end{array}$ & $\begin{array}{l}0 * * \\
.11643)\end{array}$ & $\begin{array}{r}9.56 \\
\text { (Tukey= }\end{array}$ & $\begin{array}{l}64^{* * *} \\
0.13142)\end{array}$ & $\begin{array}{r}7 . \\
\text { (Tuke) }\end{array}$ & $\begin{array}{l}23^{\star *} \\
0.12923)\end{array}$ & $\begin{array}{r}5 . \\
\text { (Tukey }\end{array}$ & $\begin{array}{l}53^{* *} \\
0.08205)\end{array}$ \\
\hline$F_{\text {plant density } \mathrm{X} \text { fertilization }}$ & & $755^{\text {ns }}$ & & $69^{\text {ns }}$ & & $7^{\text {ns }}$ & 0.5 & $73^{\text {ns }}$ & & $11^{\text {ns }}$ & & $06^{\text {ns }}$ \\
\hline
\end{tabular}

Different letters within a column indicate significant differences according to Tukey's HSD test $(\alpha=0.05)$. Significance levels: ${ }^{*} P<0.05 ;{ }^{* *} P<0.01 ;{ }^{* * *} P<0.001 ;$ ns, not significant $(P>0.05)$. 
(Table 3). Specifically, the maximum leaf dry matter per plant values (0.553 and 0.578 g plant $^{-1}$ in 2017 and 2018, respectively) were achieved in the case of the low plant density $\left(200\right.$ plants $\mathrm{m}^{-2}$ ). In regard to fertilization, the highest values $\left(0.621\right.$ and $0.652 \mathrm{~g}$ plant $^{-1}$ in 2017 and 2018 , respectively) were recorded in inorganic fertilization treatment, while the lowest were obtained in untreated plots (0.351 and 0.375 g plant $^{-1}$ in 2017 and 2018, respectively).

\section{Seed yield}

The results of the present research indicated that seed yield was influenced both by plant density and fertilization (Table 4). Concerning the effect of plant density, the seed yields recorded in low-density plots (677.3 and $602.8 \mathrm{~kg} \mathrm{ha}^{-1}$ in 2017 and 2018, respectively) were higher than in highdensity treatments (446.8 and $506.5 \mathrm{~kg} \mathrm{ha}^{-1}$ in 2017 and 2018 , respectively). In response to fertilization, the highest seed yields were achieved in inorganic fertilization (677.6 and $703.4 \mathrm{~kg} \mathrm{ha}^{-1}$ in 2017 and 2018, respectively) and compost treatments (636.4 and $619.8 \mathrm{~kg} \mathrm{ha}^{-1}$ in 2017 and 2018, respectively).

\section{Growth indices of individual plants}

Absolute growth rate (AGR) expresses the rate of change in size per unit time and constitutes the simplest growth index. The influence of the plant density and fertilization on AGR are presented in Table 5. The maximum values were achieved in the timespan between before blooming and before full flowering stage (60-75 DAS). In the treatment with the low-density, the values of AGR index were substantially higher (0.0293 and $0.0264 \mathrm{~g} \mathrm{day}^{-1}$ in 2017 and 2018, respectively) than the high-density treatment (0.0248 and $0.0220 \mathrm{~g} \mathrm{day}^{-1}$ in 2017 and 2018, respectively). The mean values of AGR provided a good evidence of the influence of fertilization treatments. Averaged over plant densities and years, the highest values were found

\begin{tabular}{|c|c|c|c|c|}
\hline \multirow[t]{4}{*}{ Fertilization } & \multicolumn{4}{|c|}{ Plant Density (plants $\mathrm{m}^{-2}$ ) } \\
\hline & 200 & 300 & 200 & 300 \\
\hline & \multicolumn{4}{|c|}{ Seed Yield $\left(\mathrm{kg} \mathrm{ha}^{-1}\right)$} \\
\hline & \multicolumn{2}{|c|}{2017} & \multicolumn{2}{|c|}{2018} \\
\hline Control & 515.2 & 344.5 & 405.3 & 371.3 \\
\hline Manure & 614.4 & 394.4 & 586.3 & 427.9 \\
\hline Compost & 779.6 & 493.2 & 670.4 & 657.8 \\
\hline Inorganic & 800.2 & 555.5 & 749.0 & 657.8 \\
\hline$F_{\text {plant density }}$ & \multicolumn{2}{|c|}{$\begin{array}{c}22.2751^{* * *} \\
(\text { Tukey }=124.77)\end{array}$} & \multicolumn{2}{|c|}{$\begin{array}{c}4.8847^{*} \\
(\text { Tukey }=131.60)\end{array}$} \\
\hline$F_{\text {fertilization }}$ & \multicolumn{2}{|c|}{$\begin{array}{c}5.5465^{* *} \\
(\text { Tukey=191.24) }\end{array}$} & \multicolumn{2}{|c|}{$\begin{array}{c}9.8899^{\star * \star} \\
(\text { Tukey } 180.46)\end{array}$} \\
\hline$F_{\text {plant density } X \text { fertilization }}$ & \multicolumn{2}{|c|}{$0.2453^{\text {ns }}$} & \multicolumn{2}{|c|}{$0.3420^{\text {ns }}$} \\
\hline
\end{tabular}

Different letters within a column indicate significant differences according to Tukey's HSD test $(\alpha=0.05)$. Significance levels: * $P<0.05 ;{ }^{* \star} P<0.01$; ${ }^{* * *} P<0.001$; ns, not significant ( $\left.>0.05\right)$. in inorganic fertilization $\left(0.0295 \mathrm{~g} \mathrm{day}^{-1}\right)$ and compost $\left(0.0291 \mathrm{~g} \mathrm{day}^{-1}\right)$. Concerning the year, the mean value was higher in 2017 (0.0271 $\left.\mathrm{g} \mathrm{day}^{-1}\right)$ than in $2018\left(0.0242 \mathrm{~g} \mathrm{day}^{-1}\right)$.

The absolute growth rate of the leaf weight (ALGR) was characterized by two phases, the first describing the leaf growth and the increase of leaf weight (positive values), while the second describing the weight loss due to the leaf withering (negative values). The averaged maximum ALGR values were obtained in the growing period between the middle and the end of vegetative stage (45-60 DAS). ALGR index was significantly affected by different plant densities, and averaged over fertilization treatments, the highest values $\left(0.0107\right.$ and $0.0114 \mathrm{~g} \mathrm{day}^{-1}$ in 2017 and 2018 , respectively) were recorded when plants subjected to lower density (Table 5). The mean value of ALGR was the greatest in the inorganic treatment $\left(0.0119 \mathrm{~g} \mathrm{day}^{-1}\right)$ followed by compost $\left(0.0110 \mathrm{~g} \mathrm{day}^{-1}\right)$, manure $\left(0.0095 \mathrm{~g} \mathrm{day}^{-1}\right)$ and control $\left(0.0068 \mathrm{~g} \mathrm{day}^{-1}\right)$.

Relative growth rate (RGR) is an index that takes into account the original difference in size of plants and, specifically, expresses growth in terms of the rate of increase in size per unit of size. RGR index was presented an initial quick increase with the highest value found between the middle and the end of vegetative growth phase (45-60 DAS), and then the index was declined with the minimum values obtained at the beginning of seed maturity (Table 6). The combined analysis of variance revealed that this index was significantly affected by both plant density and fertilization (Table 12). Averaged over fertilization treatments and years, the maximum values were 0.0667 and $0.0654 \mathrm{~g} \mathrm{~g}^{-1} \mathrm{day}^{-1}$ for low and high-density, respectively. In response to fertilization, the mean value was the highest in the inorganic treatment $\left(0.0708 \mathrm{~g} \mathrm{~g}^{-1} \mathrm{day}^{-1}\right)$ followed by compost $\left(0.0680 \mathrm{~g} \mathrm{~g}^{-1} \mathrm{day}^{-1}\right)$, manure $\left(0.0659 \mathrm{~g} \mathrm{~g}^{-1} \mathrm{day}^{-1}\right)$ and control $\left(0.0597 \mathrm{~g} \mathrm{~g}^{-1} \mathrm{day}^{-1}\right)$.

Net assimilation rate (NAR) index represents the productive efficiency of plants in capturing light, assimilating carbon dioxide and storing photo assimilate and is calculated in relation to dry matter accumulation and total leaf area of a plant. Starting from the middle of vegetative growth (45 DAS), NAR index is increased rapidly, until the period between blooming and full flowering (75 DAS), after which the rate slowed and remain almost constant during the flowering period (85 DAS). Then, the index was rapidly decreased received negative values until the end of the measurements (85-115 DAS). The negative values describing the weight loss due to the leaf withering. The maximum values were recorded in the period between before blooming and before full flowering stage (6075 DAS). NAR was significantly affected by different plant densities, and averaged over years and fertilization 
Table 5: Effect of plant density (200 and 300 plants $\mathrm{m}^{-2}$ ) and fertilization (control, manure, compost and inorganic) on absolute growth rate (AGR) and absolute leaf growth rate (ALGR) by Tukey's HSD test.

\begin{tabular}{|c|c|c|c|c|c|c|c|c|c|c|}
\hline \multirow[t]{4}{*}{ Fertilization } & \multicolumn{10}{|c|}{ Plant Density (plants $\mathrm{m}^{-2}$ ) } \\
\hline & 200 & 300 & 200 & 300 & 200 & 300 & 200 & 300 & 200 & 300 \\
\hline & \multicolumn{10}{|c|}{ Absolute Growth Rate (g day $\left.{ }^{1}\right)$} \\
\hline & \multicolumn{2}{|c|}{ 45-60 DAS } & \multicolumn{2}{|c|}{ 60-75 DAS } & \multicolumn{2}{|c|}{ 75-85 DAS } & \multicolumn{2}{|c|}{ 85-100 DAS } & \multicolumn{2}{|c|}{ 100-115 DAS } \\
\hline \multicolumn{11}{|l|}{2017} \\
\hline Control & 0.0187 & 0.0184 & 0.0228 & 0.0208 & 0.0202 & 0.0188 & 0.0030 & 0.0016 & 0.0052 & 0.0059 \\
\hline Manure & 0.0238 & 0.0201 & 0.0268 & 0.0221 & 0.0257 & 0.0209 & 0.0055 & 0.0059 & 0.0071 & 0.0048 \\
\hline Compost & 0.0283 & 0.0225 & 0.0340 & 0.0275 & 0.0330 & 0.0259 & 0.0046 & 0.0061 & 0.0087 & 0.0062 \\
\hline Inorganic & 0.0273 & 0.0235 & 0.0337 & 0.0287 & 0.0329 & 0.0275 & 0.0089 & 0.0052 & 0.0082 & 0.0083 \\
\hline$F_{\text {plant density }}$ & \multicolumn{2}{|c|}{$3.5803^{\text {ns }}$} & \multicolumn{2}{|c|}{$\begin{array}{c}4.8739^{*} \\
\text { (Tukey=0.00508) }\end{array}$} & \multicolumn{2}{|c|}{$\begin{array}{c}5.3616^{*} \\
\text { (Tukey }=0.00337 \text { ) }\end{array}$} & \multicolumn{2}{|c|}{$0.6584^{\mathrm{ns}}$} & \multicolumn{2}{|c|}{$0.9190^{\text {ns }}$} \\
\hline$F_{\text {fertilization }}$ & \multicolumn{2}{|c|}{$\begin{array}{c}3.3291^{*} \\
\text { (Tukey }=0.00541)\end{array}$} & \multicolumn{2}{|c|}{$\begin{array}{c}5.1240^{*} \\
\text { (Tukey }=0.00621)\end{array}$} & \multicolumn{2}{|c|}{$\begin{array}{c}6.3781^{* *} \\
\text { (Tukey }=0.00631)\end{array}$} & \multicolumn{2}{|c|}{$\begin{array}{c}4.3016^{*} \\
\text { (Tukey }=0.00391 \text { ) }\end{array}$} & \multicolumn{2}{|c|}{$1.3372 \mathrm{~ns}$} \\
\hline $\begin{array}{l}F_{\text {plant density } \mathrm{x} \text { fertilization }} \\
2018\end{array}$ & \multicolumn{2}{|c|}{$0.4104^{\mathrm{ns}}$} & \multicolumn{2}{|c|}{$0.2054^{\mathrm{ns}}$} & \multicolumn{2}{|c|}{$0.3584^{\text {ns }}$} & \multicolumn{2}{|c|}{$1.4245^{\mathrm{ns}}$} & \multicolumn{2}{|c|}{$0.5710^{\text {ns }}$} \\
\hline Control & 0.0175 & 0.0164 & 0.0199 & 0.0185 & 0.0156 & 0.0149 & 0.0045 & 0.0046 & 0.0043 & 0.0027 \\
\hline Manure & 0.0211 & 0.0178 & 0.0249 & 0.0199 & 0.0235 & 0.0186 & 0.0036 & 0.0045 & 0.0068 & 0.0032 \\
\hline Compost & 0.0229 & 0.0209 & 0.0302 & 0.0246 & 0.0282 & 0.0219 & 0.0046 & 0.0059 & 0.0072 & 0.0056 \\
\hline Inorganic & 0.0246 & 0.0203 & 0.0305 & 0.0249 & 0.0306 & 0.0238 & 0.0071 & 0.0057 & 0.0069 & 0.0055 \\
\hline$F_{\text {plant density }}$ & \multicolumn{2}{|c|}{$2.8435^{\text {ns }}$} & \multicolumn{2}{|c|}{$\begin{array}{c}4.7669^{*} \\
(\text { Tukey }=0.00411)\end{array}$} & $\begin{array}{r}7.0 \\
\text { (Tukey= }\end{array}$ & $\begin{array}{l}68^{*} \\
.00437)\end{array}$ & 0.1 & $57^{\text {ns }}$ & $\begin{array}{r}8.0 \\
\text { (Tukey= }\end{array}$ & $\begin{array}{l}82^{*} \\
.00168)\end{array}$ \\
\hline$F_{\text {fertilization }}$ & 2.5 & $14^{\text {ns }}$ & $\begin{array}{r}4.2 \\
\text { (Tukey= }\end{array}$ & $\left.00613^{\star}\right)$ & $\begin{array}{r}9.06 \\
\text { (Tukey= }\end{array}$ & $\begin{array}{l}18^{* *} \\
0.0576)\end{array}$ & 1.7 & $38^{\text {ns }}$ & $\begin{array}{r}3.5 \\
\text { (Tukey }\end{array}$ & $\begin{array}{l}24^{*} \\
.00239)\end{array}$ \\
\hline$F_{\text {plant density } X \text { fertilization }}$ & 0.1 & $51^{\text {ns }}$ & 0.2 & & 0.6 & $34^{\text {ns }}$ & 0.58 & $27^{\text {ns }}$ & 0.5 & $09^{\text {ns }}$ \\
\hline & & & & Absolu & Leaf Gro & vth Rate & day-1) & & & \\
\hline & $45-60 \mathrm{D}$ & & 60-75 DA & & 75-85 DA & & 85-100 D & & $100-115$ & AS \\
\hline 2017 & & & & & & & & & & \\
\hline Control & 0.0073 & 0.0059 & 0.0049 & 0.0047 & 0.0051 & 0.0042 & -0.0060 & -0.0058 & -0.0065 & -0.0058 \\
\hline Manure & 0.0108 & 0.0075 & 0.0078 & 0.0055 & 0.0078 & 0.0050 & -0.0097 & -0.0067 & -0.0091 & -0.0066 \\
\hline Compost & 0.0119 & 0.0097 & 0.0124 & 0.0083 & 0.0082 & 0.0076 & -0.0123 & -0.0097 & -0.0111 & -0.0085 \\
\hline Inorganic & 0.0127 & 0.0104 & 0.0134 & 0.0111 & 0.0120 & 0.0086 & -0.0140 & -0.0112 & -0.0121 & -0.0100 \\
\hline$F_{\text {plant density }}$ & $\begin{aligned} & 11 . \\
& \text { (Tukey }\end{aligned}$ & $\begin{array}{l}11^{* *} \\
.00210)\end{array}$ & $\begin{array}{r}12.9 \\
\text { (Tukey= }\end{array}$ & $\begin{array}{l}99^{* *} \\
.00288)\end{array}$ & 4.15 & $49^{\text {ns }}$ & $\begin{array}{r}7.1 \\
\text { (Tukey= }\end{array}$ & $\begin{array}{l}01^{*} \\
.00204)\end{array}$ & $\begin{array}{r}9.5 \\
\text { (Tukey= }\end{array}$ & $\begin{array}{l}44^{* *} \\
.00203)\end{array}$ \\
\hline$F f_{\text {ertilization }}$ & $\begin{array}{r}10.5 \\
\text { (Tukey }\end{array}$ & $\begin{array}{l}79^{* * \star} \\
.00321)\end{array}$ & $\begin{array}{r}30.6 \\
\text { (Tukey= }\end{array}$ & $\begin{array}{l}8^{\star \star \star} \\
.00316)\end{array}$ & $\begin{array}{r}6.67 \\
\text { (Tukey= }\end{array}$ & $28^{\star *}$ & $\begin{array}{r}13.2 \\
\text { (Tukey= }\end{array}$ & $\begin{array}{l}36^{\star \star \star} \\
.00361)\end{array}$ & $\begin{array}{r}11.3 \\
\text { (Tukey= }\end{array}$ & $\begin{array}{l}34^{* * *} \\
.00294)\end{array}$ \\
\hline $\begin{array}{l}F_{\text {plant density } X \text { fertilization }} \\
2018\end{array}$ & & $33^{\text {ns }}$ & 1.6 & & 0.5 & $93^{\text {ns }}$ & 0.6 & $33^{\text {ns }}$ & 0.4 & $65^{\text {ns }}$ \\
\hline Control & 0.0077 & 0.0061 & 0.0057 & 0.0052 & 0.0048 & 0.0045 & -0.0064 & -0.0063 & -0.0070 & -0.0064 \\
\hline Manure & 0.0117 & 0.0078 & 0.0082 & 0.0059 & 0.0082 & 0.0055 & -0.0100 & -0.0074 & -0.0099 & -0.0066 \\
\hline Compost & 0.0124 & 0.0100 & 0.0116 & 0.0101 & 0.0086 & 0.0077 & -0.0124 & -0.0104 & -0.0109 & -0.0095 \\
\hline Inorganic & 0.0136 & 0.0108 & 0.0154 & 0.0112 & 0.0105 & 0.0091 & -0.0144 & -0.0114 & -0.0133 & -0.0104 \\
\hline$F_{\text {plant density }}$ & $\begin{array}{r}11 . \\
\text { (Tukey }\end{array}$ & $\begin{array}{l}02^{* *} \\
.00231)\end{array}$ & $\begin{array}{r}7.5 \\
\text { (Tukey= }\end{array}$ & $\begin{array}{l}8^{*} \\
.00201)\end{array}$ & $\begin{array}{r}5.0 \\
\text { (Tukey= }\end{array}$ & $\begin{array}{l}45^{*} \\
.00103)\end{array}$ & $\begin{array}{r}6.2 \\
\text { (Tukey= }\end{array}$ & $\begin{array}{l}38^{*} \\
.00182)\end{array}$ & $\begin{array}{r}8.0 \\
\text { (Tukey= }\end{array}$ & $\begin{array}{l}70^{*} \\
.00197)\end{array}$ \\
\hline$F_{\text {fertilization }}$ & $\begin{array}{r}8.0 \\
\text { (Tukey }\end{array}$ & $18^{* *}$ & $\begin{array}{r}21.1 \\
\text { (Tukey= }\end{array}$ & $4^{* \star *}$ & $\begin{array}{r}13.30 \\
\text { (Tukey= }\end{array}$ & $\begin{array}{l}53^{* * *} \\
.00254)\end{array}$ & $\begin{array}{r}13.95 \\
\text { (Tukey= }\end{array}$ & $\begin{array}{l}00339) \\
.00 * \star\end{array}$ & $\begin{array}{r}10.0 \\
\text { (Tukey= }\end{array}$ & $\begin{array}{l}91^{* * *} \\
.00323)\end{array}$ \\
\hline$F_{\text {plant density } X \text { fertilization }}$ & 0.3 & $56^{\text {ns }}$ & 0.9 & $8^{\text {ns }}$ & 0.7 & $51^{\text {ns }}$ & 0.7 & $50^{\text {ns }}$ & 0.7 & $49^{\text {ns }}$ \\
\hline
\end{tabular}

Different letters within a column indicate significant differences according to Tukey's HSD test $(\alpha=0.05)$. Significance levels: ${ }^{*} P<0.05 ;{ }^{* *} P<0.01 ;{ }^{* *} P<0.001 ;$ ns, not significant $(P>0.05)$.

treatments, the highest value $\left(0.00301 \mathrm{~g} \mathrm{~cm}^{-2}\right.$ day $\left.^{-1}\right)$ was recorded when plants subjected to low density (Table 6). In response to fertilization, the mean value was the highest in the inorganic treatment $\left(0.00378 \mathrm{~g} \mathrm{~cm}^{-2} \mathrm{day}^{-1}\right)$ and compost $\left(0.00318 \mathrm{~g} \mathrm{~cm}^{-2} \mathrm{day}^{-1}\right)$.

Leaf area ratio (LAR) constitutes a morphological index of the plant leafiness defined as the ratio between total leaf area per plant and total weight per plant. The maximum values of LAR were achieved in the middle of vegetative stage (45 DAS), then the values declined gradually until the flowering stage (85 DAS), followed by a steeply decrease. LAR index was significantly affected by different plant densities, and averaged over years and fertilization treatments, the highest value $\left(106.35 \mathrm{~cm}^{2} \mathrm{~g}^{-1}\right)$ was recorded in low density plots (Table 7$)$. In regard 
Table 6: Effect of plant density (200 and 300 plants $\mathrm{m}^{-2}$ ) and fertilization (control, manure, compost and inorganic) on relative growth rate (RGR) and net assimilation rate (NAR) by Tukey's HSD test.

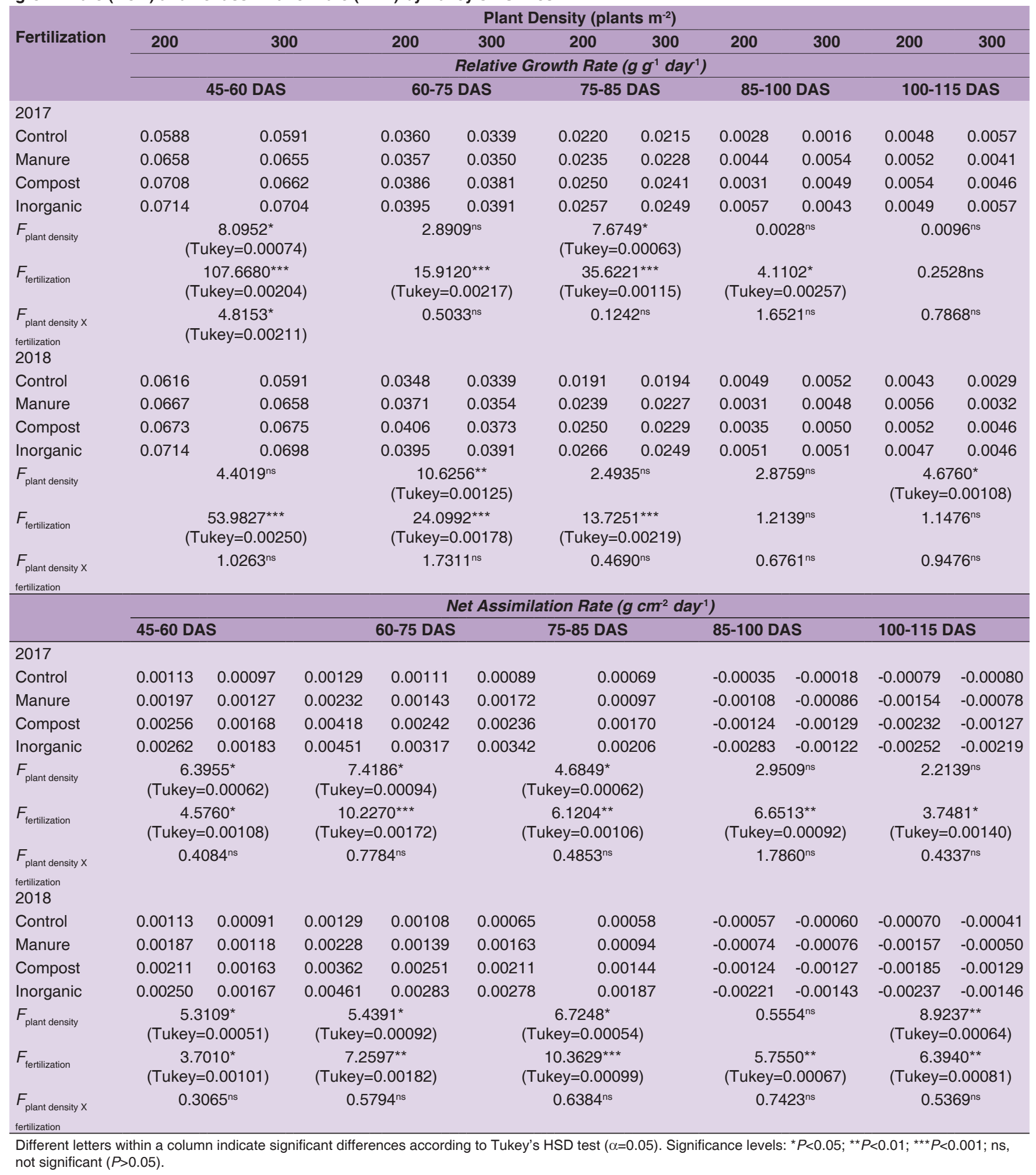

to fertilization, the highest value $\left(117.36 \mathrm{~cm}^{2} \mathrm{~g}^{-1}\right)$ was achieved in inorganic fertilization treatment, followed by compost $\left(108.29 \mathrm{~cm}^{2} \mathrm{~g}^{-1}\right)$. In response to the year, the mean value was higher in $2018\left(111.55 \mathrm{~cm}^{2} \mathrm{~g}^{-1}\right)$ than in $2017\left(93.54 \mathrm{~cm}^{2} \mathrm{~g}^{-1}\right)$.
Specific leaf area (SLA) constitutes an index of the "leafiness of the leaf" and is a measure of density or of relative thinness expressed as the ratio between total leaf area per plant and total leaf weight per plant. SLA index was significantly influenced by the fertilization and the maximum values were recorded 
Roussis, et al.

Table 7: Effect of plant density (200 and 300 plants $\mathrm{m}^{-2}$ ) and fertilization (control, manure, compost and inorganic) on leaf area ratio (LAR) and specific leaf area (SLA) by Tukey's HSD test.

\begin{tabular}{|c|c|c|c|c|c|c|c|c|c|c|c|c|}
\hline \multirow{4}{*}{ Fertilization } & \multicolumn{12}{|c|}{ Plant Density (plants $m-2$ ) } \\
\hline & 200 & 300 & 200 & 300 & 200 & 300 & 200 & 300 & 200 & 300 & 200 & 300 \\
\hline & \multicolumn{12}{|c|}{ Leaf Area Ratio (cm2 g-1) } \\
\hline & \multicolumn{2}{|c|}{45 DAS } & \multicolumn{2}{|c|}{60 DAS } & \multicolumn{2}{|c|}{75 DAS } & \multicolumn{2}{|c|}{85 DAS } & \multicolumn{2}{|c|}{100 DAS } & \multicolumn{2}{|c|}{115 DAS } \\
\hline \multicolumn{13}{|l|}{2017} \\
\hline Control & 79.27 & 74.92 & 74.90 & 65.98 & 67.88 & 64.52 & 55.87 & 52.42 & 37.04 & 33.73 & 26.50 & 27.75 \\
\hline Manure & 96.13 & 82.39 & 94.38 & 75.68 & 84.47 & 70.24 & 69.27 & 57.09 & 46.45 & 36.65 & 26.37 & 24.98 \\
\hline Compost & 104.67 & 95.03 & 100.85 & 96.89 & 86.81 & 82.19 & 72.90 & 70.37 & 52.07 & 47.74 & 24.61 & 25.70 \\
\hline Inorganic & 109.93 & 105.99 & 115.58 & 109.79 & 101.67 & 94.83 & 83.46 & 79.94 & 58.42 & 55.40 & 21.73 & 22.94 \\
\hline$F_{\text {plant density }}$ & \multicolumn{2}{|c|}{$\begin{array}{c}7.5573^{*} \\
\text { (Tukey=6.579) }\end{array}$} & \multicolumn{2}{|c|}{$\begin{array}{c}10.4129^{\star \star} \\
(\text { Tukey=8.023) }\end{array}$} & \multicolumn{2}{|c|}{$\begin{array}{c}8.1390^{*} \\
\text { (Tukey=7.037) }\end{array}$} & \multicolumn{2}{|c|}{$\begin{array}{c}7.2189^{*} \\
(\text { Tukey=4.384) }\end{array}$} & \multicolumn{2}{|c|}{$\begin{array}{c}10.3775^{\star *} \\
(\text { Tukey=4.842) }\end{array}$} & \multicolumn{2}{|c|}{$1.0246^{\mathrm{ns}}$} \\
\hline$F_{\text {fertilization }}$ & \multicolumn{2}{|c|}{$\begin{array}{c}21.5572^{\star \star \star} \\
\text { (Tukey=12.882) }\end{array}$} & \multicolumn{2}{|c|}{$\begin{array}{c}39.3318^{\star * *} \\
\text { (Tukey=14.092) }\end{array}$} & \multicolumn{2}{|c|}{$\begin{array}{c}27.8263^{* \star *} \\
(\text { Tukey }=11.681)\end{array}$} & \multicolumn{2}{|c|}{$\begin{array}{c}34.0450^{* * *} \\
\text { (Tukey }=9.279)\end{array}$} & \multicolumn{2}{|c|}{$\begin{array}{c}35.2257^{\star \star \star} \\
(\text { Tukey=7.620) }\end{array}$} & \multicolumn{2}{|c|}{$\begin{array}{c}14.1545^{\star \star \star} \\
(\text { Tukey }=1.6276)\end{array}$} \\
\hline $\begin{array}{l}F_{\text {plant density } x} \\
\text { fertilization } \\
2018\end{array}$ & 0.6 & $66^{\text {ns }}$ & 1.28 & $53^{\text {ns }}$ & 0.9 & $31^{\text {ns }}$ & 1.2 & $0^{\text {ns }}$ & 0.9 & & 1.4 & $32^{\text {ns }}$ \\
\hline Control & 96.69 & 90.21 & 88.69 & 78.52 & 82.16 & 77.42 & 69.14 & 63.72 & 43.76 & 39.15 & 31.82 & 22.62 \\
\hline Manure & 119.08 & 99.38 & 112.92 & 89.67 & 100.82 & 83.58 & 82.75 & 68.17 & 55.82 & 43.61 & 31.14 & 30.11 \\
\hline Compost & 118.97 & 114.50 & 120.93 & 112.56 & 101.75 & 97.73 & 84.61 & 82.87 & 59.54 & 58.29 & 28.73 & 30.43 \\
\hline Inorganic & 129.07 & 124.46 & 137.24 & 130.47 & 122.24 & 113.80 & 99.87 & 95.98 & 68.43 & 66.03 & 26.18 & 27.90 \\
\hline$F_{\text {plant density }}$ & $\begin{array}{r}4.5 \\
\text { (Tukey }\end{array}$ & $\begin{array}{l}58^{*} \\
=8.288)\end{array}$ & $\begin{array}{r}9.44 \\
\text { (Tukey= }\end{array}$ & $\begin{array}{l}73^{\star *} \\
18.092)\end{array}$ & $\begin{array}{r}5.4 \\
\text { (Tukey }\end{array}$ & $\begin{array}{l}58^{*} \\
14.077)\end{array}$ & $\begin{array}{r}5.7 \\
\text { (Tukey }\end{array}$ & $\begin{array}{l}23^{*} \\
6.389)\end{array}$ & $\begin{array}{r}5.5 \\
\text { (Tukey }\end{array}$ & $\begin{array}{l}8^{*} \\
4.562)\end{array}$ & 2.0 & $50^{\text {ns }}$ \\
\hline$F_{\text {fertilization }}$ & $\begin{array}{l}11.5 \\
\text { (Tukey= }\end{array}$ & $\begin{array}{l}92^{\star \star *} \\
17.474)\end{array}$ & $\begin{array}{l}29.53 \\
\text { (Tukey }\end{array}$ & $\begin{array}{l}92^{* \star *} \\
18.556)\end{array}$ & $\begin{array}{r}18.7 \\
\text { (Tukey }\end{array}$ & $\begin{array}{l}76^{* * *} \\
15.835)\end{array}$ & $\begin{array}{r}25.0 \\
\text { (Tukey }\end{array}$ & $\begin{array}{l}8^{* * *} \\
1.852)\end{array}$ & $\begin{array}{l}26 . \\
\text { (Tuke }\end{array}$ & $\begin{array}{l}4^{\star \star *} \\
9.672)\end{array}$ & $\begin{array}{r}10 . \\
\text { (Tuke }\end{array}$ & $\begin{array}{l}2 * 2^{* *} \\
2.9731)\end{array}$ \\
\hline$F_{\text {plant density } x}$ & $0.7 \varepsilon$ & $73^{\text {ns }}$ & 0.91 & $06^{\text {ns }}$ & 0.6 & $47^{\mathrm{ns}}$ & 1.1 & $7^{\text {ns }}$ & 1.2 & & 0.8 & $5^{\text {ns }}$ \\
\hline & & & & & & cific $L \epsilon$ & ea $(\mathrm{cm}$ & & & & & \\
\hline & 45 & AS & 60 & AS & 75 & AS & 85 & & 100 & AS & 115 & AS \\
\hline 2017 & & & & & & & & & & & & \\
\hline Control & 115.05 & 110.19 & 146.31 & 141.57 & 174.79 & 173.85 & 154.74 & 153.21 & 141.45 & 136.99 & 167.71 & 191.14 \\
\hline Manure & 119.33 & 117.77 & 161.10 & 152.65 & 182.08 & 178.25 & 161.07 & 157.56 & 159.17 & 149.85 & 151.73 & 170.65 \\
\hline Compost & 128.20 & 126.74 & 181.28 & 175.86 & 183.75 & 184.66 & 172.63 & 170.39 & 182.52 & 176.08 & 149.78 & 160.60 \\
\hline Inorganic & 133.88 & 133.93 & 194.74 & 193.25 & 198.86 & 194.07 & 174.58 & 177.66 & 192.11 & 187.88 & 125.78 & 138.38 \\
\hline$F_{\text {plant density }}$ & 1.98 & $69^{\text {ns }}$ & $\begin{array}{r}9.47 \\
\text { (Tukey }\end{array}$ & $\begin{array}{l}39^{* *} \\
=4.342)\end{array}$ & 1.2 & $68^{\text {ns }}$ & 0.7 & $8^{\text {ns }}$ & $\begin{array}{r}5.7 \\
\text { (Tukey }\end{array}$ & $\begin{array}{l}7^{*} \\
5.780)\end{array}$ & $\begin{array}{r}8 . \\
\text { (Tuke }\end{array}$ & $\begin{array}{l}49^{\star} \\
15.129)\end{array}$ \\
\hline$F_{\text {fertilization }}$ & $\begin{array}{r}46.1 \\
\text { (Tukey }\end{array}$ & $\begin{array}{l}02^{* * *} \\
=4.058)\end{array}$ & $\begin{array}{r}186.2 \\
\text { (Tukey }\end{array}$ & $\begin{array}{l}66^{\star \star *} \\
=7.616)\end{array}$ & $\begin{array}{r}23.0 \\
\text { (Tukey }\end{array}$ & $\begin{array}{l}36^{* \star *} \\
=7.448)\end{array}$ & $\begin{array}{r}71.3 \\
\text { (Tukey }\end{array}$ & $\begin{array}{l}8^{* \star *} \\
4.941)\end{array}$ & $\begin{array}{r}82.3 \\
\text { (Tukey= }\end{array}$ & $\begin{array}{l}2^{\star \star \star} \\
0.667)\end{array}$ & $\begin{array}{r}11.9 \\
\text { (Tukey }\end{array}$ & $\begin{array}{l}20^{\star \star \star} \\
8.739)\end{array}$ \\
\hline $\begin{array}{l}F_{\text {plant density } x} \\
\text { fertilization } \\
2018\end{array}$ & 0.5 & $58^{\text {ns }}$ & 0.76 & $78^{\text {ns }}$ & 0.4 & $17^{\mathrm{ns}}$ & 1.3 & $1^{\text {ns }}$ & 0.2 & & 0.2 & $16^{\text {ns }}$ \\
\hline Control & 115.23 & 110.59 & 148.14 & 141.68 & 174.19 & 173.85 & 155.92 & 151.82 & 140.19 & 137.53 & 167.80 & 195.76 \\
\hline Manure & 121.49 & 118.22 & 158.56 & 152.77 & 183.72 & 179.89 & 163.18 & 158.17 & 157.69 & 150.54 & 150.00 & 169.21 \\
\hline Compost & 128.39 & 127.96 & 177.39 & 178.18 & 186.61 & 181.80 & 171.75 & 166.41 & 180.90 & 178.14 & 151.05 & 159.73 \\
\hline Inorganic & 130.98 & 131.05 & 194.74 & 191.50 & 196.30 & 195.88 & 179.22 & 178.34 & 189.21 & 188.31 & 128.69 & 139.55 \\
\hline$F_{\text {plant density }}$ & 1.9 & $67^{\text {ns }}$ & 2.67 & $69^{\text {ns }}$ & 1.6 & $30^{\text {ns }}$ & 4.4 & & 1.27 & & $\begin{array}{r}7.3 \\
\text { (Tukey }\end{array}$ & $\begin{array}{l}15^{\star} \\
6.377)\end{array}$ \\
\hline$F_{\text {fertilization }}$ & $\begin{array}{r}30.4 \\
\text { (Tukey }\end{array}$ & $\begin{array}{l}74^{* * *} \\
=5.840)\end{array}$ & $\begin{array}{r}93.43 \\
\text { (Tukey }\end{array}$ & $\begin{array}{l}80^{* * *} \\
=8.947)\end{array}$ & $\begin{array}{r}25.1 \\
\text { (Tukey }\end{array}$ & $\begin{array}{l}76^{* * *} \\
=7.013)\end{array}$ & $\begin{array}{r}34.9 \\
\text { (Tukey }\end{array}$ & $\begin{array}{l}7 * * * \\
7.449)\end{array}$ & $\begin{array}{r}58.9 \\
\text { (Tukey= }\end{array}$ & $\begin{array}{l}9^{* * *} \\
1.171)\end{array}$ & $\begin{array}{r}10.0 \\
\text { (Tukey }\end{array}$ & $\begin{array}{l}23^{* * *} \\
20.239)\end{array}$ \\
\hline $\begin{array}{l}F_{\text {plant density } x} \\
\text { fertilization }\end{array}$ & 0.5 & $38^{\text {ns }}$ & 0.53 & $50^{\text {ns }}$ & 0.4 & $03^{\text {ns }}$ & 0.3 & $4^{\text {ns }}$ & $0.1 s$ & & 0.5 & $3^{\text {ns }}$ \\
\hline
\end{tabular}

Different letters within a column indicate significant differences according to Tukey's HSD test $(\alpha=0.05)$. Significance levels: ${ }^{*} P<0.05 ;{ }^{* *} P<0.01 ;{ }^{* * *} P<0.001 ;$ ns, not significant $(P>0.05)$.

between blooming and full flowering stage (75 DAS) (Table 7). The greatest values (196.47 and $196.09 \mathrm{~cm}^{2} \mathrm{~g}^{-1}$ in 2017 and 2018, respectively) were recorded in inorganic fertilization treatment, while the lowest were obtained in untreated plots (174.32 and $174.02 \mathrm{~cm}^{2} \mathrm{~g}^{-1}$ in 2017 and 2018, respectively).
Leaf weight ratio (LWR) is an index of the plant leafiness on a dry weight basis defined as the ratio between total leaf weight per plant and total weight per plant. The maximum LWR values were obtained in the middle of vegetative growth stage (45 DAS), and then were gradually decreased 
until the end of measurements. According to the combined analysis of variance, LWR index was significantly affected by the different plant densities and fertilization treatments. In response to plant density, the highest values $(0.7825$ and $0.9321 \mathrm{~g} \mathrm{~g}^{-1}$ in 2017 and 2018, respectively) obtained when plants subjected to low-density (200 plants $\mathrm{m}^{-2}$ ) (Table 8). Additionally, the highest LWR index was achieved in inorganic fertilization with the values being 0.8057 (18\% higher than control) and $0.9671 \mathrm{~g} \mathrm{~g}^{-1}(17 \%$ higher than control) in 2017 and 2018, respectively. Concerning the year, the mean value was higher in $2018\left(0.9033 \mathrm{~g} \mathrm{~g}^{-1}\right)$ than in $2017\left(0.7562 \mathrm{~g} \mathrm{~g}^{-1}\right)$.

Specific leaf weight (SLW) is the index of "leaf leafiness" on a dry weight basis obtained using the relation total leaf area per plant to total leaf weight per plant. SLW was significantly affected by the different plant density and fertilization treatments and the maximum value was reached in the middle of vegetative growth stage (45 DAS). Regarding plant density, averaged over years and fertilization treatments, the lowest value $\left(0.0081 \mathrm{~g} \mathrm{~cm}^{-2}\right)$ was recorded when plants subjected to low density (Table 8). The mean value of SLW reduced to the inorganic fertilization treatment, and the mean values in the separate treatments were as follows: control: 0.0089 , manure: 0.0084 , compost: 0.0078 and inorganic: $0.0075 \mathrm{~g} \mathrm{~cm}^{-2}$.

\section{Growth indices of the crop stand}

Crop growth rate (CGR) constitutes an agricultural productivity index of land expressed as the growth in relation to plant biomass produced per unit area and its increase can be plotted as a logarithmic or exponential curve. According to the results of this study, the dynamics of CGR were similar to those of AGR and this could be explained by the fact that the AGR index is the slope of CGR's curve. As with AGR, the maximum values of CGR were obtained in the growing period between before blooming and before full flowering stage (60-75 DAS). CGR index was significantly influenced by the different plant densities, and averaged over years and fertilization treatments, the highest value $\left(7.0132 \mathrm{~g} \mathrm{~m}^{-2} \mathrm{day}^{-1}\right)$ was recorded under the high density (Table 9). Regarding the fertilization treatments, the maximum values were found in inorganic fertilization (7.6355 and $6.7785 \mathrm{~g} \mathrm{~m}^{-2} \mathrm{day}^{-1}$ in 2017 and 2018, respectively) and compost (7.5303 and $6.7109 \mathrm{~g}$ $\mathrm{m}^{-2} \mathrm{day}^{-1}$ in 2017 and 2018, respectively).

Leaf area index (LAI) is defined as the total leaf area of the crop over a unit of the land. The effect of the plant density and fertilization treatments on LAI are presented in Table 10. The maximum values were recorded at flowering stage (85 DAS). LAI was significantly higher $\left(2.253 \mathrm{~m}^{2} \mathrm{~m}^{-2}\right)$ in the high-density $\left(300\right.$ plants $\left.\mathrm{m}^{-2}\right)$ than in low-density $\left(200\right.$ plants $\left.\mathrm{m}^{-2}\right)$ plots $\left(1.905 \mathrm{~m}^{2} \mathrm{~m}^{-2}\right)$ during the experimental periods. The mean values of LAI were higher in the inorganic treatment $\left(2.757 \mathrm{~m}^{2} \mathrm{~m}^{-2}\right)$ and compost $\left(2.389 \mathrm{~m}^{2} \mathrm{~m}^{-2}\right)$ followed by manure $\left(1.789 \mathrm{~m}^{2} \mathrm{~m}^{-2}\right)$ and control $\left(1.380 \mathrm{~m}^{2} \mathrm{~m}^{-2}\right)$.

Leaf area duration (LAD) constitutes a quantitative expression of green leaf retention over time. According to the analysis of variance, the LAD was significantly affected both by plant density and fertilization with the maximum values found in the period between flowering stage and the first days of seed formation (85-100 DAS) (Table 11). In regard to plant density, the highest values (29.03 days) obtained when plants subjected to high-density ( 300 plants $\mathrm{m}^{-2}$ ). The highest $\mathrm{LAD}$ values, averaged over years and plant density treatments, were achieved in inorganic fertilization and compost with the values being 36.07 and 31.25 days.

Biomass duration (BMD) constitutes a measurement of biomass persistence with time. The effects of plant density and fertilization on BMD were significant during the cultivation periods and the highest values were achieved in the middle of seed formation period (100115 DAS) (Tables 11 and 12). Averaged over years and fertilization treatments, the value of BMD in low-density treatments (20.29 days) was higher than in high-density plots (17.38 days). Concerning the effect of fertilization, the maximum values were found in inorganic fertilization (21.47 days) and compost (20.91 days). In response to the year, the mean value was higher in the first year (19.98 days) than in the second one (17.69 days).

\section{DISCUSSION}

Growth analysis of N. sativa revealed significant relationships between growth rate and seed yield both the single plant and crop stand level. Specifically, the AGR, ALGR, RGR and CGR had a strong positive correlation with seed yield $(r=0.8751, p<0.001 ; r=0.8506, p<0.001, r=0.6273 ; p<0.001$ and $r=0.4307, p=0.002$, respectively). These results are in line with other studies presenting a significant association between growth rate and yield in several crop species, such as wheat (Sugár et al., 2017), maize (Bullock et al., 1993), sugar beet (Hoffmann and Kluge-Severin, 2011) and oilseed rape (Hunková et al., 2011). The effects of plant density on seed yield was significant in all experimental years. The seed yield was higher in the plant density of 200 plants $\mathrm{m}^{-2}$ with the averaging value being $34 \%$ higher in comparison to 300 plants $\mathrm{m}^{-2}$. Mollafilabi et al. (2010) observed that the highest seed yield was obtained in the plant density of 180 plants $\mathrm{m}^{-2}\left(809 \mathrm{~kg} \mathrm{ha}^{-1}\right)$ and an increase to 240 plants $\mathrm{m}^{-2}$ reduced the yield by $38 \%$. Regarding the fertilization, there was a significant increase in seed yield with the increase of nitrogen fertilization rates. The seed 
Roussis, et al.

Table 8: Effect of plant density (200 and 300 plants $\mathrm{m}^{-2}$ ) and fertilization (control, manure, compost and inorganic) on leaf weight ratio (LWR) and specific leaf weight (SLW) by Tukey's HSD test.

\begin{tabular}{|c|c|c|c|c|c|c|c|c|c|c|c|c|}
\hline \multirow{4}{*}{ Fertilization } & \multicolumn{12}{|c|}{ Plant Density (plants $m^{-2}$ ) } \\
\hline & 200 & 300 & 200 & 300 & 200 & 300 & 200 & 300 & 200 & 300 & 200 & 300 \\
\hline & \multicolumn{12}{|c|}{ Leaf Weight Ratio $\left(g g^{-1}\right)$} \\
\hline & \multicolumn{2}{|c|}{45 DAS } & \multicolumn{2}{|c|}{60 DAS } & \multicolumn{2}{|c|}{75 DAS } & \multicolumn{2}{|c|}{85 DAS } & \multicolumn{2}{|c|}{100 DAS } & \multicolumn{2}{|c|}{115 DAS } \\
\hline \multicolumn{13}{|l|}{2017} \\
\hline Control & 0.6889 & 0.6794 & 0.5117 & 0.4659 & 0.3883 & 0.3709 & 0.3609 & 0.3422 & 0.2618 & 0.2460 & 0.1582 & 0.1453 \\
\hline Manure & 0.8051 & 0.6998 & 0.5855 & 0.4955 & 0.4640 & 0.3938 & 0.4299 & 0.3624 & 0.2917 & 0.2443 & 0.1739 & 0.1468 \\
\hline Compost & 0.8159 & 0.7492 & 0.5569 & 0.5507 & 0.4722 & 0.4449 & 0.4222 & 0.4127 & 0.2852 & 0.2710 & 0.1645 & 0.1606 \\
\hline Inorganic & 0.8201 & 0.7913 & 0.5929 & 0.5682 & 0.5111 & 0.4883 & 0.4776 & 0.4498 & 0.3037 & 0.2946 & 0.1736 & 0.1665 \\
\hline$F_{\text {plant density }}$ & \multicolumn{2}{|c|}{$\begin{array}{c}9.6158^{* *} \\
\text { (Tukey }=0.04690)\end{array}$} & \multicolumn{2}{|c|}{$\begin{array}{c}8.7567^{* *} \\
\text { (Tukey }=0.04100)\end{array}$} & \multicolumn{2}{|c|}{$\begin{array}{c}9.4626^{* *} \\
\text { (Tukey }=0.02644)\end{array}$} & \multicolumn{2}{|c|}{$\begin{array}{c}9.5413^{* *} \\
\text { (Tukey }=0.02701)\end{array}$} & \multicolumn{2}{|c|}{$\begin{array}{c}12.2979^{* *} \\
\text { (Tukey }=0.01939)\end{array}$} & \multicolumn{2}{|c|}{$\begin{array}{c}15.3256^{* *} \\
\text { (Tukey=0.00905) }\end{array}$} \\
\hline$F_{\text {fertilization }}$ & \multicolumn{2}{|c|}{$\begin{array}{c}9.6894^{* \star \star} \\
\text { (Tukey }=0.08265)\end{array}$} & \multicolumn{2}{|c|}{$\begin{array}{c}7.4810^{* *} \\
(\text { Tukey }=0.06788)\end{array}$} & \multicolumn{2}{|c|}{$\begin{array}{c}20.4544^{\star \star \star} \\
(\text { Tukey }=0.05328)\end{array}$} & \multicolumn{2}{|c|}{$\begin{array}{c}21.7382^{* * *} \\
\text { (Tukey=0.04882) }\end{array}$} & \multicolumn{2}{|c|}{$\begin{array}{c}9.5442^{* * *} \\
(\text { Tukey=0.02380) }\end{array}$} & \multicolumn{2}{|c|}{$\begin{array}{c}2.4802^{*} \\
\text { (Tukey=0.01800) }\end{array}$} \\
\hline $\begin{array}{l}F_{\text {plant density } x} \\
\text { fertilization } \\
2018\end{array}$ & & $1.5686^{\mathrm{ns}}$ & & $1.6335^{\mathrm{ns}}$ & $1.1715^{\mathrm{ns}}$ & $15^{\mathrm{ns}}$ & 1.63 & $57^{\text {ns }}$ & 2.0 & $09^{\text {ns }}$ & 2.4 & $02^{\text {ns }}$ \\
\hline Control & 0.8392 & 0.8153 & 0.5985 & 0.5537 & 0.4718 & 0.4451 & 0.4435 & 0.4199 & 0.3119 & 0.2845 & 0.1899 & 0.1721 \\
\hline Manure & 0.9800 & 0.8397 & 0.7117 & 0.5864 & 0.5489 & 0.4644 & 0.5072 & 0.4312 & 0.3539 & 0.2892 & 0.2080 & 0.1785 \\
\hline Compost & 0.9242 & 0.8936 & 0.6816 & 0.6328 & 0.5445 & 0.5368 & 0.4914 & 0.4982 & 0.3280 & 0.3269 & 0.1913 & 0.1909 \\
\hline Inorganic & 0.9848 & 0.9495 & 0.7043 & 0.6811 & 0.6219 & 0.5805 & 0.5583 & 0.5378 & 0.3609 & 0.3506 & 0.2047 & 0.2003 \\
\hline$F_{\text {plant density }}$ & $\begin{array}{r}5.2 \\
\text { (Tukey= }\end{array}$ & $\begin{array}{l}.02^{\star} \\
.05310)\end{array}$ & $\begin{array}{r}9.66 \\
\text { (Tukey= }\end{array}$ & $\begin{array}{l}70^{\star *} \\
.05366)\end{array}$ & $\begin{array}{r}6.26 \\
\text { (Tukey= }\end{array}$ & $\begin{array}{l}10^{*} \\
.03543)\end{array}$ & 3.62 & Ons & $\begin{array}{r}11.7 \\
\text { (Tukey= }\end{array}$ & $\begin{array}{l}143^{\star *} \\
0.02494)\end{array}$ & $\begin{array}{r}7.3 \\
\text { (Tukey= }\end{array}$ & $\begin{array}{l}36^{*} \\
.01197)\end{array}$ \\
\hline$F f_{\text {ertilization }}$ & $\begin{array}{r}5.2 \\
\text { (Tukey= }\end{array}$ & $\begin{array}{l}80^{*} \\
.11095)\end{array}$ & $\begin{array}{r}6.3 \\
\text { (Tukey= }\end{array}$ & $\begin{array}{l}60^{\star *} \\
.09373)\end{array}$ & $\begin{array}{r}14.04 \\
\text { (Tukey= }\end{array}$ & $\begin{array}{l}68^{* * *} \\
.07143)\end{array}$ & $\begin{array}{r}10.77 \\
\text { (Tukey= }\end{array}$ & $\begin{array}{l}97^{\star * \star} \\
.04775)\end{array}$ & $\begin{array}{r}9.78 \\
\text { (Tukey= }\end{array}$ & $\begin{array}{l}25^{\star \star *} \\
0.04127)\end{array}$ & $\begin{array}{r}3.3 \\
\text { (Tukey= }\end{array}$ & $\begin{array}{l}80^{*} \\
.01706)\end{array}$ \\
\hline $\begin{array}{l}\mathrm{F}_{\text {plant density } x} \\
\text { fertilization }\end{array}$ & 1.2 & $42^{\text {ns }}$ & 1.3 & $36^{\text {ns }}$ & 1.04 & $23^{\text {ns }}$ & 1.35 & $\mathrm{O}^{\text {ns }}$ & $\begin{array}{r}3.4 \\
\text { (Tukey= }\end{array}$ & $\begin{array}{l}192^{*} \\
0.03204)\end{array}$ & 1.9 & $70^{\text {ns }}$ \\
\hline & & & & & Spe & ific Leaf & Veight (g & $\left.m^{-2}\right)$ & & & & \\
\hline & 45 & AS & 60 & AS & 75 & AS & 85 & AS & 100 & DAS & 115 & DAS \\
\hline 2017 & & & & & & & & & & & & \\
\hline Control & 0.0087 & 0.0091 & 0.0068 & 0.0071 & 0.0057 & 0.0058 & 0.0065 & 0.0065 & 0.0071 & 0.0073 & 0.0060 & 0.0053 \\
\hline Manure & 0.0084 & 0.0085 & 0.0062 & 0.0066 & 0.0055 & 0.0056 & 0.0062 & 0.0064 & 0.0063 & 0.0067 & 0.0066 & 0.0059 \\
\hline Compost & 0.0078 & 0.0079 & 0.0055 & 0.0057 & 0.0054 & 0.0054 & 0.0058 & 0.0059 & 0.0055 & 0.0057 & 0.0067 & 0.0063 \\
\hline Inorganic & 0.0074 & 0.0075 & 0.0051 & 0.0052 & 0.0050 & 0.0052 & 0.0057 & 0.0056 & 0.0052 & 0.0053 & 0.0080 & 0.0072 \\
\hline$F_{\text {plant density }}$ & 2.8 & $21^{\text {ns }}$ & $\begin{array}{r}10.1 \\
\text { (Tukey= }\end{array}$ & $\begin{array}{l}31^{* *} \\
.00017)\end{array}$ & 0.67 & $92^{\text {ns }}$ & 0.2 & $32^{\text {ns }}$ & $\begin{array}{r}7.5 \\
\text { (Tukey= }\end{array}$ & $\begin{array}{l}00^{*} \\
0.00018)\end{array}$ & $\begin{array}{r}7.8 \\
\text { (Tukey= }\end{array}$ & $\begin{array}{l}02^{*} \\
.00057)\end{array}$ \\
\hline$F_{\text {fertilization }}$ & $\begin{array}{r}50.8 \\
\text { (Tukey= }\end{array}$ & $\begin{array}{l}21^{* * *} \\
.00036)\end{array}$ & $\begin{array}{r}178.0 \\
\text { (Tukey= }\end{array}$ & $\begin{array}{l}192^{\star \star *} \\
.00029)\end{array}$ & $\begin{array}{c}20.15 \\
\text { (Tukey= }\end{array}$ & $\begin{array}{l}09^{* \star *} \\
.00023)\end{array}$ & $\begin{array}{r}58.26 \\
\text { (Tukey= }\end{array}$ & $\begin{array}{l}13^{* * *} \\
.00020)\end{array}$ & $\begin{array}{r}95.1 \\
\text { (Tukey= }\end{array}$ & $\begin{array}{l}56^{* \star *} \\
0.00041)\end{array}$ & $\begin{array}{r}12.9 \\
\text { (Tukey= }\end{array}$ & $\begin{array}{l}47^{* \star *} \\
.00075)\end{array}$ \\
\hline $\begin{array}{l}F_{\text {plant density } x} \\
\text { fertilization } \\
2018\end{array}$ & $0.6 s$ & $01^{\text {ns }}$ & 1.0 & $62^{\text {ns }}$ & 0.52 & $83^{\text {ns }}$ & $0.8 s$ & $19^{\text {ns }}$ & 0.5 & $67^{\text {ns }}$ & 0.1 & $89^{\text {ns }}$ \\
\hline Control & 0.0087 & 0.0090 & 0.0068 & 0.0071 & 0.0057 & 0.0058 & 0.0064 & 0.0066 & 0.0072 & 0.0073 & 0.0060 & 0.0052 \\
\hline Manure & 0.0082 & 0.0085 & 0.0063 & 0.0066 & 0.0054 & 0.0056 & 0.0061 & 0.0063 & 0.0063 & 0.0067 & 0.0067 & 0.0059 \\
\hline Compost & 0.0078 & 0.0078 & 0.0056 & 0.0056 & 0.0054 & 0.0055 & 0.0058 & 0.0060 & 0.0055 & 0.0056 & 0.0066 & 0.0063 \\
\hline Inorganic & 0.0076 & 0.0076 & 0.0051 & 0.0052 & 0.0051 & 0.0051 & 0.0055 & 0.0056 & 0.0053 & 0.0053 & 0.0079 & 0.0072 \\
\hline$F_{\text {plant density }}$ & 2.06 & $43^{\text {ns }}$ & 3.1 & $21^{\text {ns }}$ & 1.39 & $13^{\text {ns }}$ & $\begin{array}{r}4.6 \\
\text { (Tukey= }\end{array}$ & $\begin{array}{l}57^{*} \\
.00014)\end{array}$ & 1.4 & $50^{\text {ns }}$ & $\begin{array}{r}7.1 \\
\text { (Tukey= }\end{array}$ & $\begin{array}{l}61^{*} \\
.00076)\end{array}$ \\
\hline$F_{\text {fertilization }}$ & $\begin{array}{r}32.5 \\
\text { (Tukey= }\end{array}$ & $\begin{array}{l}14^{\star * *} \\
.00039)\end{array}$ & $\begin{array}{r}75.9 \\
\text { (Tukey= }\end{array}$ & $\begin{array}{l}26^{\star \star \star} \\
.00036)\end{array}$ & $\begin{array}{c}22.43 \\
\text { (Tukey= }\end{array}$ & $\begin{array}{l}48^{* * \star} \\
.00021)\end{array}$ & $\begin{array}{r}33.50 \\
\text { (Tukey= }\end{array}$ & $\begin{array}{l}72^{* * \star} \\
.00029)\end{array}$ & $\begin{array}{r}56.1 \\
\text { (Tukey= }\end{array}$ & $\begin{array}{l}17^{\star * \star} \\
0.00045)\end{array}$ & $\begin{array}{r}11.1 \\
\text { (Tukey= }\end{array}$ & $\begin{array}{l}10^{* \star *} \\
.00078)\end{array}$ \\
\hline $\begin{array}{l}F_{\text {plant density X }} \\
\text { fertilization }\end{array}$ & 0.75 & $00^{\text {ns }}$ & 0.5 & $03^{\text {ns }}$ & 0.28 & $99^{\text {ns }}$ & 0.32 & $35^{\text {ns }}$ & 0.35 & $17^{\mathrm{ns}}$ & 0.1 & $49^{\text {ns }}$ \\
\hline
\end{tabular}

yield was lowest in control and manure treatments with a significant increase in compost and inorganic fertilization treatments. According to several authors, the crops fertilized with inorganic fertilizers gave greater yields since these fertilizers contained soluble inorganic nitrogen with quick availability for crop species resulting in higher yields (Bilalis et al., 2018; Kakabouki et al., 2018). In our study, despite the differences in accumulated growing degree days (ADD) between the studied growing periods (1417.3 and 1685.2 ADD in 2017 and 2018, respectively), there were 
Roussis, et al.

Table 9: Effect of plant density (200 and 300 plants $\mathrm{m}^{-2}$ ) and fertilization (control, manure, compost and inorganic) on crop growth rate (CGR) by Tukey's HSD test.

\begin{tabular}{|c|c|c|c|c|c|c|c|c|c|c|}
\hline \multirow{4}{*}{ Fertilization } & \multicolumn{10}{|c|}{ Plant Density (plants $m^{-2}$ ) } \\
\hline & 200 & 300 & 200 & 300 & 200 & 300 & 200 & 300 & 200 & 300 \\
\hline & \multicolumn{10}{|c|}{ Crop Growth Rate $\left(\mathrm{g} \mathrm{m}^{2}\right.$ day $\left.^{-1}\right)$} \\
\hline & \multicolumn{2}{|c|}{ 45-60 DAS } & \multicolumn{2}{|c|}{ 60-75 DAS } & \multicolumn{2}{|c|}{ 75-85 DAS } & \multicolumn{2}{|c|}{ 85-100 DAS } & \multicolumn{2}{|c|}{ 100-115 DAS } \\
\hline \multicolumn{11}{|l|}{2017} \\
\hline Control & 3.7460 & 5.5320 & 4.5693 & 6.2410 & 4.0470 & 5.6483 & 0.5953 & 0.4747 & 1.0497 & 1.7650 \\
\hline Manure & 4.7523 & 6.0397 & 5.3647 & 6.6390 & 5.1470 & 6.2770 & 1.0963 & 1.7687 & 1.4250 & 1.4317 \\
\hline Compost & 5.6667 & 6.7443 & 6.8037 & 8.2569 & 6.6010 & 7.7513 & 0.9283 & 1.8523 & 1.7493 & 1.8623 \\
\hline Inorganic & 5.4710 & 7.0593 & 6.6690 & 8.6020 & 6.5760 & 8.2573 & 1.7737 & 1.5623 & 1.6340 & 2.4810 \\
\hline $\mathrm{F}_{\text {plant density }}$ & \multicolumn{2}{|c|}{$\begin{array}{c}9.8040^{\star *} \\
(\text { Tukey }=1.0088)\end{array}$} & \multicolumn{2}{|c|}{$\begin{array}{c}9.4130^{\star *} \\
(\text { Tukey=1.2533) }\end{array}$} & \multicolumn{2}{|c|}{$\begin{array}{c}7.4801^{*} \\
(\text { Tukey=1.3019) }\end{array}$} & \multicolumn{2}{|c|}{$2.0201^{\mathrm{ns}}$} & \multicolumn{2}{|c|}{$2.2845^{\text {ns }}$} \\
\hline$F_{\text {fertilization }}$ & \multicolumn{2}{|c|}{$2.8119^{\text {ns }}$} & \multicolumn{2}{|c|}{$\begin{array}{c}4.6524^{*} \\
(\text { Tukey }=1.7232)\end{array}$} & \multicolumn{2}{|c|}{$\begin{array}{c}5.7611^{* *} \\
(\text { Tukey }=1.6339)\end{array}$} & \multicolumn{2}{|c|}{$\begin{array}{c}4.9793^{\star} \\
\text { (Tukey=0.94195) }\end{array}$} & \multicolumn{2}{|c|}{$1.2744^{\text {ns }}$} \\
\hline $\begin{array}{l}F_{\text {plant density } x \text { fertilization }} \\
2018\end{array}$ & \multicolumn{2}{|c|}{$0.1175^{\text {ns }}$} & \multicolumn{2}{|c|}{$0.0760^{\text {ns }}$} & \multicolumn{2}{|c|}{$0.0820^{\text {ns }}$} & \multicolumn{2}{|c|}{$1.6267^{\mathrm{ns}}$} & \multicolumn{2}{|c|}{$0.5756^{\text {ns }}$} \\
\hline Control & 3.5073 & 4.9283 & 3.9817 & 5.5597 & 3.1083 & 4.4733 & 0.9063 & 1.3750 & 0.8547 & 0.8193 \\
\hline Manure & 4.2140 & 5.3540 & 4.9680 & 5.9566 & 4.7120 & 5.5850 & 0.7076 & 1.3686 & 1.3520 & 0.9510 \\
\hline Compost & 4.5680 & 6.2577 & 6.0377 & 7.3840 & 5.6386 & 6.5703 & 0.9237 & 1.7583 & 1.4460 & 1.6747 \\
\hline Inorganic & 4.9163 & 6.0920 & 6.0907 & 7.4663 & 6.1140 & 7.1690 & 1.4113 & 1.7246 & 1.3816 & 1.6676 \\
\hline $\mathrm{F}_{\text {plant density }}$ & \multicolumn{2}{|c|}{$\begin{array}{c}10.7592^{* *} \\
\text { (Tukey }=0.86946)\end{array}$} & \multicolumn{2}{|c|}{$\begin{array}{c}6.9725^{\star} \\
\text { (Tukey = 1.1655) }\end{array}$} & \multicolumn{2}{|c|}{$\begin{array}{c}5.3968^{*} \\
\text { (Tukey = 1.0253) }\end{array}$} & \multicolumn{2}{|c|}{$\begin{array}{c}8.8697^{* *} \\
\text { (Tukey }=0.39246)\end{array}$} & \multicolumn{2}{|c|}{$0.0135^{\text {ns }}$} \\
\hline$F_{\text {fertilization }}$ & \multicolumn{2}{|c|}{$2.1074^{\text {ns }}$} & \multicolumn{2}{|c|}{$\begin{array}{c}3.8455^{*} \\
\text { (Tukey = 1.5893) }\end{array}$} & \multicolumn{2}{|c|}{$\begin{array}{c}7.5611^{* *} \\
\text { (Tukey = 1.3929) }\end{array}$} & \multicolumn{2}{|c|}{$1.4980^{\text {ns }}$} & \multicolumn{2}{|c|}{$\begin{array}{c}4.1266^{*} \\
\text { (Tukey }=0.64269)\end{array}$} \\
\hline$F_{\text {plant density } X \text { fertilization }}$ & & & 0.0 & $99^{\text {ns }}$ & 0.0 & $2^{\text {ns }}$ & & & & \\
\hline
\end{tabular}

Different letters within a column indicate significant differences according to Tukey's HSD test $(\alpha=0.05)$. Significance levels: ${ }^{*} P<0.05 ;{ }^{* *} P<0.01 ;{ }^{* *} P<0.001$; ns, not significant $(P>0.05)$

Table 10: Effect of plant density (200 and 300 plants $\mathrm{m}^{-2}$ ) and fertilization (control, manure, compost and inorganic) on leaf area index (LAI) by Tukey's HSD test.

\begin{tabular}{|c|c|c|c|c|c|c|c|c|c|c|c|c|}
\hline \multirow{4}{*}{ Fertilization } & \multicolumn{12}{|c|}{ Plant Density (plants $m^{2}$ ) } \\
\hline & 200 & 300 & 200 & 300 & 200 & 300 & 200 & 300 & 200 & 300 & 200 & 300 \\
\hline & \multicolumn{12}{|c|}{ Leaf Area Index $\left(\mathrm{m}^{2} \mathrm{~m}^{2}\right)$} \\
\hline & \multicolumn{2}{|c|}{45 DAS } & \multicolumn{2}{|c|}{60 DAS } & \multicolumn{2}{|c|}{75 DAS } & \multicolumn{2}{|c|}{85 DAS } & \multicolumn{2}{|c|}{100 DAS } & \multicolumn{2}{|c|}{115 DAS } \\
\hline \multicolumn{13}{|l|}{2017} \\
\hline Control & 0.314 & 0.436 & 0.718 & 0.932 & 1.116 & 1.515 & 1.145 & 1.527 & 0.792 & 1.005 & 0.608 & 0.902 \\
\hline Manure & 0.407 & 0.446 & 1.073 & 1.101 & 1.639 & 1.726 & 1.702 & 1.757 & 1.218 & 1.228 & 0.745 & 0.895 \\
\hline Compost & 0.470 & 0.563 & 1.309 & 1.549 & 2.014 & 2.321 & 2.173 & 2.532 & 1.626 & 1.844 & 0.832 & 1.077 \\
\hline Inorganic & 0.462 & 0.593 & 1.411 & 1.764 & 2.246 & 2.736 & 2.378 & 2.963 & 1.817 & 2.179 & 0.744 & 1.002 \\
\hline$F_{\text {plant density }}$ & \multicolumn{2}{|c|}{$\begin{array}{c}8.6777^{\star \star} \\
(\text { Tukey }=0.0819)\end{array}$} & \multicolumn{2}{|c|}{$\begin{array}{c}5.4549^{*} \\
(\text { Tukey }=0.2058)\end{array}$} & \multicolumn{2}{|c|}{$\begin{array}{c}5.4531^{*} \\
\text { (Tukey }=0.3071)\end{array}$} & \multicolumn{2}{|c|}{$\begin{array}{c}5.8572^{*} \\
\text { (Tukey }=0.33203)\end{array}$} & \multicolumn{2}{|c|}{$3.9488^{\mathrm{ns}}$} & \multicolumn{2}{|c|}{$\begin{array}{c}10.2169^{* *} \\
\text { (Tukey }=0.1480)\end{array}$} \\
\hline$F_{\text {fertilization }}$ & \multicolumn{2}{|c|}{$\begin{array}{c}5.0260^{\star} \\
\text { (Tukey }=0.1469)\end{array}$} & \multicolumn{2}{|c|}{$\begin{array}{c}14.7306^{* * *} \\
(\text { Tukey }=0.3804)\end{array}$} & \multicolumn{2}{|c|}{$\begin{array}{c}14.3009^{* * *} \\
\text { (Tukey=0.5782) }\end{array}$} & \multicolumn{2}{|c|}{$\begin{array}{c}17.7728^{* * *} \\
(\text { Tukey }=0.6139)\end{array}$} & \multicolumn{2}{|c|}{$\begin{array}{c}23.9874^{* * *} \\
\text { (Tukey }=0.4151)\end{array}$} & \multicolumn{2}{|c|}{$1.2993^{\mathrm{ns}}$} \\
\hline$F_{\text {plant density } x}$ & \multicolumn{2}{|c|}{$0.4126^{\mathrm{ns}}$} & \multicolumn{2}{|c|}{$0.5730^{\text {ns }}$} & \multicolumn{2}{|c|}{$0.3975^{\text {ns }}$} & \multicolumn{2}{|c|}{$0.5864^{\mathrm{ns}}$} & \multicolumn{2}{|c|}{$0.5156^{\text {ns }}$} & \multicolumn{2}{|c|}{$0.1731^{\mathrm{ns}}$} \\
\hline \multicolumn{13}{|l|}{$\begin{array}{l}\text { fertilization } \\
2018\end{array}$} \\
\hline Control & 0.335 & 0.468 & 0.774 & 0.988 & 1.208 & 1.620 & 1.231 & 1.617 & 0.839 & 1.075 & 0.650 & 0.964 \\
\hline Manure & 0.438 & 0.476 & 1.129 & 1.157 & 1.759 & 1.823 & 1.834 & 1.861 & 1.297 & 1.290 & 0.785 & 0.941 \\
\hline Compost & 0.470 & 0.609 & 1.309 & 1.654 & 2.031 & 2.503 & 2.169 & 2.682 & 1.609 & 2.028 & 0.836 & 1.144 \\
\hline Inorganic & 0.489 & 0.610 & 1.518 & 1.822 & 2.433 & 2.847 & 2.606 & 3.081 & 1.922 & 2.292 & 0.804 & 1.058 \\
\hline$F_{\text {plant density }}$ & \multicolumn{2}{|c|}{$\begin{array}{c}7.9482^{*} \\
\text { (Tukey=0.0883) }\end{array}$} & \multicolumn{2}{|c|}{$4.2930^{\text {ns }}$} & \multicolumn{2}{|c|}{$\begin{array}{c}4.7020^{*} \\
(\text { Tukey }=0.3204)\end{array}$} & \multicolumn{2}{|c|}{$4.2587^{\mathrm{ns}}$} & & $0446^{\mathrm{ns}}$ & & $\begin{array}{l}924^{* *} \\
=0.1619)\end{array}$ \\
\hline$F_{\text {fertilization }}$ & $\begin{array}{r}3 . \\
\text { (Tuke) }\end{array}$ & $\begin{array}{l}91^{*} \\
0.1261)\end{array}$ & $\begin{array}{r}10.6 \\
\text { (Tukey }\end{array}$ & $\begin{array}{l}56^{* * *} \\
.4416)\end{array}$ & $\begin{array}{r}11.6 \\
\text { (Tuke) }\end{array}$ & $\begin{array}{l}69^{* * *} \\
.6488)\end{array}$ & $\begin{array}{r}13.6 \\
\text { (Tukey }\end{array}$ & $\begin{array}{l}7^{* * *} \\
6968)\end{array}$ & $\begin{array}{r}16 \\
\text { (Tuk€ }\end{array}$ & $\begin{array}{l}6976^{* * *} \\
y=0.5216)\end{array}$ & & $175^{\text {ns }}$ \\
\hline$F_{\text {plant density } x \text { fertilization }}$ & 0.3 & $87^{\text {ns }}$ & 0.4 & $16^{\text {ns }}$ & 0.3 & $17^{\text {ns }}$ & 0.4 & $5^{\text {ns }}$ & & $5696^{\text {ns }}$ & & $933^{\text {ns }}$ \\
\hline
\end{tabular}

Different letters within a column indicate significant differences according to Tukey's HSD test $(\alpha=0.05)$. Significance levels: ${ }^{\star} P<0.05 ;{ }^{\star \star} P<0.01 ;{ }^{\star \star \star} P<0.001 ;$ ns, not significant $(P>0.05)$.

no significant differences on seed yield and the harvesting took place at almost the same time (122 and 125 DAS in 2017 and 2018, respectively).

The growth rates (AGR, ALGR, RGR, CGR) were positively affected by fertilization and the results were in accordance with the increase in the dry matter of leaves and above-ground parts and seed yield. As with plant density, the increase of density contributed to the decrease of the studied growth rates of individual plants (AGR, ALGR, RGR). Intra- and inter- plant competition constitute one of the most important stresses affecting biomass yield, 
Table 11: Effect of plant density (200 and 300 plants $\mathrm{m}^{-2}$ ) and fertilization (control, manure, compost and inorganic) on leaf area duration (LAD) and biomass duration (BMD) by Tukey's HSD test.

\begin{tabular}{|c|c|c|c|c|c|c|c|c|c|c|}
\hline \multirow{4}{*}{ Fertilization } & \multicolumn{10}{|c|}{ Plant Density (plants $\mathrm{m}^{-2}$ ) } \\
\hline & 200 & 300 & 200 & 300 & 200 & 300 & 200 & 300 & 200 & 300 \\
\hline & \multicolumn{10}{|c|}{ Leaf Area Duration (days) } \\
\hline & \multicolumn{2}{|c|}{ 45-60 DAS } & \multicolumn{2}{|c|}{ 60-75 DAS } & \multicolumn{2}{|c|}{ 75-85 DAS } & \multicolumn{2}{|c|}{ 85-100 DAS } & \multicolumn{2}{|c|}{ 100-115 DAS } \\
\hline \multicolumn{11}{|l|}{2017} \\
\hline Control & 7.74 & 10.26 & 13.76 & 18.35 & 11.31 & 15.21 & 14.53 & 18.99 & 10.50 & 14.30 \\
\hline Manure & 11.10 & 11.59 & 20.35 & 21.19 & 16.70 & 17.41 & 21.90 & 22.39 & 14.73 & 15.92 \\
\hline Compost & 13.34 & 11.84 & 24.93 & 29.02 & 20.94 & 24.27 & 28.49 & 32.83 & 18.43 & 21.91 \\
\hline Inorganic & 14.05 & 17.68 & 27.42 & 33.75 & 23.12 & 28.49 & 31.46 & 38.56 & 19.20 & 23.87 \\
\hline$F_{\text {plant density }}$ & \multicolumn{2}{|c|}{$\begin{array}{c}6.2791^{*} \\
(\text { Tukey=1.124) }\end{array}$} & \multicolumn{2}{|c|}{$\begin{array}{c}5.4874^{\star} \\
(\text { Tukey=3.849) }\end{array}$} & \multicolumn{2}{|c|}{$\begin{array}{c}5.6816^{*} \\
(\text { Tukey=3.007) }\end{array}$} & \multicolumn{2}{|c|}{$\begin{array}{c}5.0640 * \\
(\text { Tukey=4.015) }\end{array}$} & \multicolumn{2}{|c|}{$6.8039^{*}$} \\
\hline$F_{\text {fertilization }}$ & $\begin{array}{r}11 . \\
\text { (Tuke }\end{array}$ & $11.6690^{\star * *}$ & \multicolumn{2}{|c|}{$\begin{array}{c}14.5530^{\star \star *} \\
(\text { Tukey=7.166) }\end{array}$} & \multicolumn{2}{|c|}{$\begin{array}{c}16.0914^{\star * *} \\
(\text { Tukey=5.947) }\end{array}$} & \multicolumn{2}{|c|}{$\begin{array}{c}20.4091^{\star \star *} \\
(\text { Tukey=7.690) }\end{array}$} & \multicolumn{2}{|c|}{$\begin{array}{c}11.3740 * * * \\
(\text { Tukey }=5.441)\end{array}$} \\
\hline $\begin{array}{l}F_{\text {plant density } x \text { fertilization }} \\
2018\end{array}$ & \multicolumn{2}{|c|}{$0.5151^{\mathrm{ns}}$} & \multicolumn{2}{|c|}{$0.4559^{\text {ns }}$} & \multicolumn{2}{|c|}{$0.4870^{\text {ns }}$} & \multicolumn{2}{|c|}{$0.5616^{\mathrm{ns}}$} & \multicolumn{2}{|c|}{$0.3471^{\mathrm{ns}}$} \\
\hline Control & 8.32 & 10.91 & 14.86 & 19.55 & 12.19 & 16.19 & 15.53 & 20.19 & 11.17 & 15.29 \\
\hline Manure & 11.75 & 12.24 & 21.66 & 22.34 & 17.97 & 18.42 & 23.49 & 23.63 & 15.62 & 16.73 \\
\hline Compost & 13.34 & 16.97 & 25.05 & 31.18 & 21.00 & 25.93 & 28.34 & 35.32 & 18.33 & 23.79 \\
\hline Inorganic & 15.06 & 18.24 & 29.63 & 35.02 & 25.20 & 29.64 & 33.96 & 40.29 & 20.45 & 25.12 \\
\hline$F_{\text {plant density }}$ & $\begin{array}{r}5 . \\
\text { (Tuke }\end{array}$ & $\begin{array}{l}3^{*} \\
2.139)\end{array}$ & \multicolumn{2}{|c|}{$\begin{array}{c}4.5560 * \\
(\text { Tukey=4.125) }\end{array}$} & 4. & $9^{\text {ns }}$ & 4.1 & $2^{\text {ns }}$ & $\begin{array}{r}6 . \\
\text { (Tuke }\end{array}$ & $\begin{array}{l}8^{*} \\
3.366)\end{array}$ \\
\hline$F_{\text {fertilization }}$ & $\begin{array}{r}8 . \\
\text { (Tuke }\end{array}$ & $\begin{array}{l}4^{* *} \\
4.563)\end{array}$ & $\begin{array}{l}11.3 \\
\text { (Tuke }\end{array}$ & $\begin{array}{l}6^{* \star *} \\
3.158)\end{array}$ & $\begin{array}{l}12.7 \\
\text { (Tuke }\end{array}$ & $\begin{array}{l}2^{* \star *} \\
.714)\end{array}$ & $\begin{array}{r}14.9 \\
\text { (Tuke }\end{array}$ & $\begin{array}{l}5^{* * *} \\
9.122)\end{array}$ & $\begin{array}{r}8.4 \\
\text { (Tuke }\end{array}$ & $\begin{array}{l}3^{* \star} \\
6.520)\end{array}$ \\
\hline$F_{\text {plant density } \mathrm{x} \text { fertilization }}$ & & $1^{\text {ns }}$ & 0.3 & $3^{\text {ns }}$ & 0.3 & $8^{\text {ns }}$ & 0.4 & $2^{\text {ns }}$ & & $1^{\text {ns }}$ \\
\hline & & & & & lass $D c$ & ion (da & & & & \\
\hline & 45- & DAS & $60-7$ & JAS & $75-\varepsilon$ & DAS & $85-1$ & DAS & $100-1$ & DAS \\
\hline 2017 & & & & & & & & & & \\
\hline Control & 4.77 & 4.68 & 9.53 & 9.20 & 9.19 & 8.74 & 15.69 & 14.74 & 16.62 & 15.57 \\
\hline Manure & 5.42 & 4.60 & 11.27 & 9.50 & 10.94 & 9.15 & 19.03 & 16.00 & 20.44 & 17.21 \\
\hline Compost & 5.99 & 5.10 & 13.20 & 10.85 & 13.18 & 10.73 & 22.86 & 18.80 & 24.36 & 20.19 \\
\hline Inorganic & 5.76 & 5.01 & 12.76 & 11.03 & 12.83 & 11.03 & 22.79 & 19.27 & 24.71 & 20.78 \\
\hline$F_{\text {plant density }}$ & & $8^{\text {ns }}$ & 3.4 & $5^{\text {ns }}$ & & $9^{\text {ns }}$ & 4.1 & $2^{\text {ns }}$ & & $5^{\text {ns }}$ \\
\hline$F_{\text {fertilization }}$ & & $6^{\text {ns }}$ & 2.3 & $4^{\text {ns }}$ & $\begin{array}{r}3 . \\
\text { (Tuke }\end{array}$ & $2.446)$ & $\begin{array}{r}3 . \varepsilon^{(T u k e} \\
\text { (Tuk }\end{array}$ & $\begin{array}{l}74^{*} \\
5.752)\end{array}$ & $\begin{array}{r}4 . \\
\text { (Tuke }\end{array}$ & $\begin{array}{l}2^{*} \\
6.259)\end{array}$ \\
\hline $\begin{array}{l}F_{\text {plant density } X \text { fertilization }} \\
2018\end{array}$ & & $8^{\text {ns }}$ & 0.2 & $7^{\text {ns }}$ & & $4^{\text {ns }}$ & 0.2 & $2^{\text {ns }}$ & & $1^{\mathrm{ns}}$ \\
\hline Control & 4.27 & 4.17 & 8.58 & 8.19 & 8.10 & 7.69 & 13.86 & 13.20 & 14.84 & 14.02 \\
\hline Manure & 4.73 & 4.06 & 10.03 & 8.43 & 9.85 & 8.14 & 17.01 & 14.16 & 18.16 & 15.03 \\
\hline Compost & 5.09 & 4.64 & 11.14 & 9.89 & 11.27 & 9.66 & 19.63 & 16.85 & 20.95 & 18.13 \\
\hline Inorganic & 5.18 & 4.37 & 11.52 & 9.58 & 11.65 & 9.58 & 20.65 & 16.87 & 22.23 & 18.14 \\
\hline$F_{\text {plant density }}$ & & $7^{\text {ns }}$ & $2 . \subseteq$ & $7^{\text {ns }}$ & 3.7 & $4^{\text {ns }}$ & 3.7 & $3^{\text {ns }}$ & 3.7 & $5^{\text {ns }}$ \\
\hline$F_{\text {fertilization }}$ & & $6^{\text {ns }}$ & $1 . c$ & $2^{\text {ns }}$ & 2. & $4^{\text {ns }}$ & $\begin{array}{r}3.5 \\
\text { (Tuke }\end{array}$ & $\begin{array}{l}52^{*} \\
5.211)\end{array}$ & $\begin{array}{r}3 . \\
\text { (Tuke }\end{array}$ & $\begin{array}{l}0^{*} \\
5.594)\end{array}$ \\
\hline$F_{\text {plant density } \mathrm{X} \text { fertilization }}$ & & $3^{\text {ns }}$ & 0.1 & $3^{\text {ns }}$ & 0.2 & $3^{\text {ns }}$ & 0.2 & $9^{\text {ns }}$ & $0.2^{2}$ & $0^{\text {ns }}$ \\
\hline
\end{tabular}

ns, not significant $(p>0.05)$.

crop yield as well as economic profitability. Increasing plant density per unit ground surface area increases both types of competition for production inputs, including solar radiation, nutrients, and water. On the contrary, low densities lead to inefficiency of these inputs (Al-Suhaibani et al., 2013).

Highest AGR and CGR during the vegetative and reproductive growth stages were calculated in plots received the inorganic fertilizer. The progressive increases in the dry mass accumulation of plants, for inorganic fertilization, probably, related to a high availability of nutrients and a greater efficiency in absorption and accumulation of nutrients for formation of new tissues (Bullock et al.,
1993). The increase in the growth rate of N. sativa when fertilized with the highest rate of nitrogen (inorganic fertilization), may be associated with the increase in the number of leaves and leaf area. Accordingly, it has been found that the nitrogen deficiency could increase cell-wall peroxidase activity (bulk and spatial) in the elongation zone, presenting a reciprocal action between leaf growth cessation and enzyme activity (Djakovic and Jovanovic, 2003). Plant density had no significant effect on AGR and CGR at reproductive stage; however, higher AGR values were found in the low-density plants compared to AGR values in the high density ( 300 plants $\left.\mathrm{m}^{-2}\right)$ plots, in contrast to higher CGR values obtained at high density than at low density (200 plants $\mathrm{m}^{-2}$ ). In general, plant density affects 


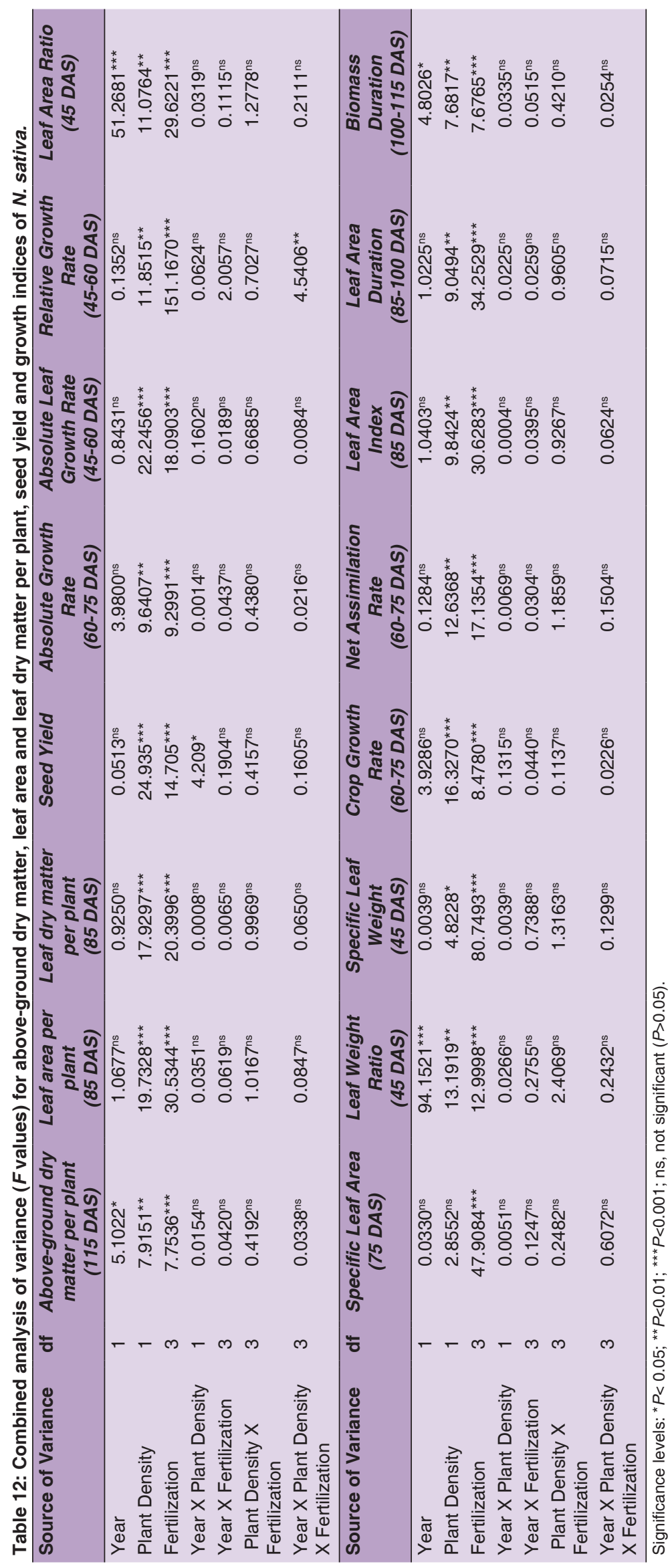


plant architecture, changes growth and developmental patterns and influences the production and partition of carbohydrates (Casal et al., 1985).

RGR is a crucial parameter consisting of one of the most ecologically important indices of plant growth. It represents the plant efficiency to produce tissues from the existing ones (Hunt, 1990). In the current study, the two peaks of maximum for RGR during vegetative growth phase and minimum during the seed maturity could give an explanation of the significant increase and decrease in plant biomass throughout these periods, respectively. The RGR decreased with the increase in plant age due to the progressive increase of non-assimilator tissues. The low RGR values derived throughout the reproductive stage coincided with the diversion of photosynthates into the seed maturation (El-Darier et al., 2002).

NAR and LAR contributed differently to RGR depending both on plant density and fertilization. There were significant positive correlations between RGR and its components, NAR and LAR $(r=0.6724, p<0.001$ and $r=0.7122, p<0.001$, respectively). The two components determined $75.32 \%(p<0.001)$ of the variance in RGR index. The CGR had a positive and significant correlation with its two components, LAI and NAR $(r=0.8597$, $p<0.001$ and $r=0.5796, p<0.001$, respectively), which together explained $81.53 \%(p<0.001)$ of the variance in CGR index. NAR and LAR were equally affected the RGR at the individual plant level; while, at crop level, the influence of LAI on CGR was pivotal and that of NAR only secondary. A study of Poorter (1989) on interspecific variation in RGR demonstrated that $80-90 \%$ of an innately higher RGR was clarified by a higher LAR and only $10-20 \%$ by a higher NAR. In general, inherently fast-growing species tend to have a higher LAR than slow-growing ones (Poorter, 1989).

LAR is determined as SLA times LWR, represents useful leaf area for photosynthesis and is the ratio of photosynthesizing to respiring material within the plant (Hunt, 1990). Both SLA and LAR constitute a ratio of leaf area to mass and both decrease in value, as leaves become thicker with higher chlorophyll and photosynthetic cells concentration and hence have greater photosynthetic capacities than thinner leaves (Craufurd et al., 1999). SLA index was only influenced by the fertilization and the maximum values were recorded in inorganic fertilization plots between blooming and full flowering stage (75 DAS). Plants with high SLA values are described by high nitrogen concentrations, high carbon dioxide rates and nitrogen uptake per unit leaf and root mass, respectively; and a high photosynthesis capacity (per unit leaf dry weight or nitrogen) (Amanullah, 2015). There was a negative and very strong correlation between SLA and SLW $(r=0.9968$, $p<0.001)$. SLA increased with the passage of time, while on the other hand SLW decreased. The increase in the leaf area per plant and reduction in the leaf dry matter per plant increased SLA index; in contrast, increase in the leaf dry matter and decrease in the leaf area per plant increased SLW. Plant species with high SLA and low SLW are better adapted in resource-rich environments, while species with low SLA and high SLW demonstrated better adaptation in resource-rich environments (Li et al., 2005).

Increase in above-ground dry matter due to the application of higher rates of nitrogen have been related with higher LAI and LAD values. In the present study, the maximum $L A I$ and $L A D$ values were recorded in inorganic fertilization at flowering stage (85 DAS) and throughout the period between flowering stage and the first days of seed formation (85-100 DAS), respectively. Supply with higher nitrogen rates generally lead to higher leaf area which, subsequently, results in greater absorption of light and further carbon fixation. The percentage of light intercepted increased gradually with an increase in plant density. The increase in light interception in high density treatment $(300$ plants $\mathrm{m}^{-2}$ ) was probably due to the increase of LAI at high densities (Amanullah et al., 2007). Seed yield had a positive and strong correlation with LAI and LAD $(r=0.5911$, $p<0.001$ and $r=0.6011, p<0.001$, respectively). According to several researchers (Pepó and Novák, 2016; Tagliapietra et al. 2018), there is a close positive association between LAI and seed yield. Positive correlations between leaf area duration and seed yield have been also observed in several crops, such as wheat (Sugár et al., 2017) and oilseed rape (Hunková et al., 2011). The significant relationship between LAI and BMD $(r=0.6553, p<0.001)$ and between LAD and BMD $(r=0.6593, p<0.001)$ indicated the importance of size and duration of the leaf area in biomass formation, while the correlation between AGR and BMD $(r=0.7496$, $p<0.001)$ pointed to the importance of growth rate in biomass formation.

\section{CONCLUSIONS}

The results of the present study confirmed that growth parameters and yield at both the single plant and crop stand level were affected by both plant density and fertilization. The highest leaf area per plant, leaf dry matter per plant and seed yield were observed following the low-plant density (200 plants $\mathrm{m}^{-2}$ ) and the application of the inorganic fertilizer. In terms of dry matter per plant, there were significant differences among the fertilization treatments with the highest value obtained under inorganic fertilization. Concerning the growth rates, AGR, ALGR, RGR, and NAR were influenced by the different plant 
densities and fertilization treatments, with the highest values found in the low-plant density and inorganic fertilization plots. The highest CGR was obtained under high-plant density and inorganic fertilization. Significantly higher LAR and LWR were also recorded when plants subjected to low-density and inorganic fertilization. SLA was only affected by fertilization with the higher values found in inorganic fertilized treatments. Moreover, the increase of plant density increased the SLW, while the application of fertilizers declined this index. LAI, LAD and BMD increased with the increase of plant density and application of inorganic fertilizer. As a conclusion, plant densities higher than 200 plants $\mathrm{m}^{-2}$ lead to higher crop growth, but lower growth of individual plants and decreased seed yield, while the application of inorganic fertilizers increases crop growth and yield as these fertilizers contain higher levels of nitrogen with high solubility and therefore quick availability for the crop than the organic fertilizers.

\section{Authors' contributions}

All authors contributed equally to the design and implementation of the research, to the analysis of the results and to the writing of the manuscript.

\section{REFERENCES}

Ahmad, A., A. Husain, M. Mujeeb, S. A. Khan, A. K. Najmi, N. A. Siddique, Z. A. Damanhouri and F. Anwar. 2013. A review on therapeutic potential of Nigella sativa: A miracle herb. Asian Pac. J. Trop. Biomed. 3: 337-352.

Al-Suhaibani, N., S. El-Hendawy and U. Schmidhalter. 2013. Influence of varied plant density on growth, yield and economic return of drip irrigated faba bean (Vicia faba L.). Turk. J. Field Crops 18(2): 185-197.

Amanullah. 2015. Specific leaf area and specific leaf weight in small grain crops wheat, rye, barley, and oats differ at various growth stages and NPK Source. J. Plant Nutr. 38(11): 1694-1708.

Amanullah, M. J. Hassan, K. Nawab and A. Ali. 2007. Response of specific leaf area, leaf area index and ratio of maize (Zea mays L.) to plant density, rate and timing of $\mathrm{N}$ application. World App Sci J. 2(3): 235-243.

Ashraf, M., Q. Ali and Z. Iqbal. 2006. Effect of nitrogen application rate on the content and composition of oil, essential oil and minerals in black cumin (Nigella sativa L.) seeds. J. Sci. Food Agric. 86: 871-876.

Banerjee, S., S. Padhye, A. Azmi, Z. Wang, P. A. Philip, O. Kucuk, F. H. Sarkar and R.M. Mohammad. 2010. Review on molecular and therapeutic potential of thymoquinone in cancer. Nutr. Cancer 62(7): 938-946.

Bilalis, D., M. Krokida, I. Roussis, P. Papastylianou, I. Travlos, N. Cheimona, and A. Dede. 2018. Effects of organic and inorganic fertilization on yield and quality of processing tomato (Lycopersicon esculentum Mill.). Folia Hortic. 30(2): 321-332.

Bullock, D. G., F. W. Simons, I. M. Chung and G. I. Johnson. 1993. Growth analysis of corn grown with or without starter fertilizer. Crop Sci. 33: 112-117.

Casal, J. J., V. A. Deregibus and R. A. Sánchez. 1985. Variations in tiller dynamics and morphology in Lolium multiflorum Lam. Vegetative and reproductive plants as affected by differences in red/far-red irradiation. Ann. Bot. 56: 533-559.

Craufurd, P. Q., T. R. Wheeler, R. H. Elli, R. J. Summerfiled and J. H. Williams. 1999. Effect of temperature and water deficit on water-use efficiency, carbon isotope discrimination, and specific leaf area in peanut. Crop Sci. 39: 136-142.

Dai, J. L., W. J. Li, W. Tang, D. M. Zhang, Z. H. Li, H. Q. Lu, A. E. Eneji and $\mathrm{H}$. Z. Dong. 2015. Manipulation of dry matter accumulation and partitioning with plant density in relation to yield stability of cotton under intensive management. Field Crops Res. 180: $207-215$.

Djakovic, T. and Z. Jovanovic. 2003. The role of cell wall peroxidase in the inhibition of leaf and fruit growth. Bulg. J. Plant Physiol. 2003: 264-272.

Dordas, C. 2009. Biomass, nitrogen and phosphorus accumulation, partitioning and remobilization as affected by $\mathrm{N}$ and $\mathrm{P}$ fertilization and source-sink relations. Eur. J. Agron. 30: 129-139.

EC 834/2007. 2007. (EC) No. 834/2007 of 28 June 2007 on Organic Production and Labeling of Organic Products and Repealing Regulation (EEC) No. 2092/91. The Council of the European Union, Europe.

El-Darier, S., M. Hemada and L. Sadek. 2002. Dry matter distribution and growth analysis in soybeans under natural agricultural conditions. Pak. J. Biol. Sci. 5(5): 545-549.

Ghaderi, F. A., A. Soltani and H. R. Sadeghipour. 2008. Cardinal temperatures of germination in medicinal pumpkin (Curcubita pepo var. pepo var. styriaca), borago (Borago officinalis L.) and black cumin (Nigella sativa L.). Asian J. Plant Sci. 7: 574-578.

Hiltbrunner, J., B. Streit and M. Liedgens. 2007. Are seeding densities an opportunity to increase grain yield of winter wheat in a living mulch of white clover? Field Crops Res. 102: 163-171.

Hoffmann, C. M. and S. Kluge-Severin. 2011. Growth analysis of autumn and spring sown sugar beet. Eur. J. Agron. 34: 1-9.

Hunková, E., M. Živčak and K. Olšovská. 2011. Leaf area duration of oilseed rape (Brassica napus subsp. napus) varieties and hybrids and its relationship to selected growth and productivity parameters. J Cent. Eur. Agric. 12(1): 1-15.

Hunt, R. 1990. Basic growth analysis Plant Growth Analysis for Beginners. Unwin Hyman, London, UK.

Kakabouki, I. P., D. Hela, I. Roussis, P. Papastylianou, A. F. Sestras and D. J. Bilalis. 2018. Influence of fertilization and soil tillage on nitrogen uptake and utilization efficiency of quinoa crop (Chenopodium quinoa Willd.). J. Soil Sci. Plant Nutr. 18(1): 220235.

Lambers, H., H. Poorter and M.M.I. Van Vuuren. 1998. Inherent Variation in Plant Growth. Backhuys Publisher, Leiden, The Netherlands.

Li, Y., D. A. Johnson, S. U. Yongzhong, C. U. I. Jianyuan and T. Zhang. 2005. Specific leaf area and leaf dry matter content of plants growing in sand dunes. Bot. Bull. Acad. Sin. (Taipei) 46: $127-134$.

Mollafilabi, A., H. Moodi, M. H. Rashed and M. Kafi. 2010. Effect of plant density and nitrogen on yield and yield components of black cumin (Nigella sativa L.). Acta Hortic. 853: 115-126.

Montenegro, R. O., S. Magnitskiy, L. P. Moreno and A. E. Darghan. 2017. Growth of Jatropha curcas L. fertilized with nitrogen and potassium under conditions of dry tropical forest of Colombia. Agron. Colomb. 35(2): 223-231.

Pepó, P. and A. Novák. 2016. Correlation between photosynthetic traits and yield in sunflower. Plant Soil Environ. 62(7): 335-340.

Piras, A., A. Rosa, B. Marongiu, S. Porcedda, D. Falconieri, M. A. Dessi, 
B. Ozcelik and U. Koca. 2013. Chemical composition and in vitro bioactivity of the volatile and fixed oils of Nigella sativa L. extracted by supercritical carbon dioxide. Ind. Crops Prod. 46: 317-323.

Poorter, H. 1989. Interspecific variation in relative growth rate: on ecological causes and physiological consequences. In: Lambers, H., M. L. Cambridge, H. Konings and T. L. Pons, editors. Causes and Consequences of Variation in Growth Rate and Productivity of Higher Plants, SPB Academic Publishing, The Netherlands, pp. $45-68$.

Razavi, B. M. and H. Hosseinzadeh. 2014. A review of the effects of Nigella sativa L. and its constituent, thymoquinone, in metabolic syndrome. J. Endocrinol. Invest. 37(11): 1031-1040.

Riaz, M., M. Syed and F. M. Chaudhary. 1996. Chemistry of the medicinal plants of the genus Nigella. Hamdard Med. 39: 40-45.

Roussis, I., I. Travlos, D. Bilalis and I. Kakabouki. 2017. Influence of seed rate and fertilization on yield and yield components of Nigella sativa L. under Mediterranean semi-arid conditions. AgroLife Sci. J. 6(1): 218-223.

Sugár, E., Z. Berzsenyi, P. Bónis and T. Árendás. 2017. Growth analysis of winter wheat cultivars as affected by nitrogen fertilization. Wachstumsanalyse von winterweizensorten in abhängigkeit von stickstoffdüngung. Die bodenkultur. J. Land Manag. Food Environ. 68(1): 57-70.

Tagliapietra, E. L., N. A. Streck, T. S. M. Da Rocha, G. L. Richter, M. R. Da Silva, J. C. Cera, J. V. C. Guedes and A. J. Zanon. 2018. Optimum Leaf Area Index to Reach Soybean Yield Potential in Subtropical Environment. Agron. J. 110: 932-938.

Tuncturk, R., M. Tuncturk and V. Ciftci. 2012. The effects of varying nitrogen doses on yield and some yield components of black cumin (Nigella sativa L.). Adv. Environ. Biol. 6(2): 855-858.

Weraduwage, S. M., J. Chen, F. C. Anozie, A. Morales, S. E. Weise and T. D. Sharkey. 2015. The relationship between leaf area growth and biomass accumulation in Arabidopsis thaliana. Front. Plant Sci. 6: 167.

Yao, H. S., Y. L. Zhang, X. P. Yi, Y. Y. Hu, H. H. Luo, L. Gou and W.F. Zhang. 2015. Plant density alters nitrogen partitioning among photosynthetic components, leaf photosynthetic capacity and photosynthetic nitrogen use efficiency in field-grown cotton. Field Crops Res. 184: 39-49.

Zhang, H. and S. Flottmann. 2016. Seed yield of canola (Brassica napus L.) is determined primarily by biomass in a high-yielding environment. Crop Pasture Sci. 67: 369-380. 